\title{
Dynamic Behavior of Tied-Arch Bridges under the Action of Moving Loads
}

\author{
Paolo Lonetti, Arturo Pascuzzo, and Alessandro Davanzo \\ Department of Civil Engineering, University of Calabria, Via Pietro Bucci, Cubo 39-B, Rende, 87030 Cosenza, Italy \\ Correspondence should be addressed to Paolo Lonetti; paolo.lonetti@unical.it
}

Received 27 January 2016; Accepted 7 April 2016

Academic Editor: Xiao-Qiao He

Copyright (c) 2016 Paolo Lonetti et al. This is an open access article distributed under the Creative Commons Attribution License, which permits unrestricted use, distribution, and reproduction in any medium, provided the original work is properly cited.

\begin{abstract}
The dynamic behavior of tied-arch bridges under the action of moving load is investigated. The main aim of the paper is to quantify, numerically, dynamic amplification factors of typical kinematic and stress design variables by means of a parametric study developed in terms of the structural characteristics of the bridge and moving loads. The basic formulation is developed by using a finite element approach, in which refined schematization is adopted to analyze the interaction between the bridge structure and moving loads. Moreover, in order to evaluate, numerically, the influence of coupling effects between bridge deformations and moving loads, the analysis focuses attention on usually neglected nonstandard terms in the inertial forces concerning both centripetal acceleration and Coriolis acceleration. Sensitivity analyses are proposed in terms of dynamic impact factors, in which the effects produced by the external mass of the moving system on the dynamic bridge behavior are evaluated.
\end{abstract}

\section{Introduction}

The tied-arch bridge represents a valid solution in the field of medium spans, since it combines both structural and aesthetic advantages [1]. The structure consists, basically, of an arch and a girder, which are connected through internal supports. In particular, the bridge is based on an arch entirely above the girder and a tie chord at girder level, which eliminates the horizontal thrust of the arch. The structural scheme of tied-arch bridges makes it possible to have light structural components, involving low values of ratios between dead and live loads. However, due to the new developments in rapid transportation systems, the allowable train speed range and the loads involved by the moving system are much larger than the ones observed in the past. Consequently, the moving system may influence the bridge vibrations, since the external mass applied to the bridge is quite comparable to the one involved by the structural or nonstructural elements of the bridge. Moreover, existing codes on arch bridges do not provide any explicit relationships to quantify numerically the dynamic amplification factors involved in stress or displacement variables of the bridge components. To this end, accurate investigations are needed to describe the interaction between external moving system and bridge vibrations and, consequently, to estimate, correctly, dynamic impact factors of typical design bridge variables. In the literature, several works have been developed with the analysis of bridges travelled by trains, mainly for beam and girder bridges. In this framework, earlier studies were devoted to investigate the vibrations of simply supported beams travelled by moving load or mass at constant speed, providing both analytical and numerical solutions [2-5]. However, advances in computer and computational technologies made it possible to develop more accurate models and, at the same time, to extend investigations on the dynamic behavior of complex structures, such as long-span bridges [6-9]. Moreover, further issues such as track irregularities and mechanical properties of vehicles were properly taken into account in [10-13]. Recently, in order to assess the safety of new bridges against human and nature hazards, the moving load problem was examined introducing additional complexities arising from damage phenomena involved in the structural elements $[14,15]$ and, in this framework, dynamic amplification factors were properly identified. At the same time, new analyses, which combine refined models and probabilistic methodologies, were developed to assess the safety of existing bridges for high-speed traffic loads $[16,17]$. However, despite the great interest on the subject, 
there are comparatively few papers on the dynamic response of arch structures under the action of moving loads. Among these studies, Chatterjee and Datta [18] have investigated the dynamic behavior of upper-deck arch bridges travelled by a single moving load by means of a mixed approach based on continuum and lumped mass descriptions. Wu and Chiang [19] have used a numerical model developed in the framework of the FE approach to analyze in-plane bending vibrations of a uniform circular arch under the action of a moving load. Huang [20] has performed dynamic impact analyses of 3D half-through arch bridges with refined schematization of both bridge and vehicle. Ju and Lin [21] have presented an advanced numerical model to investigate the vibration characteristics of steel tied-arch bridges traversed by high-speed trains. In particular, they have proposed two simple design criteria to predict the train-bridge resonance effects, whose results were validated by means of numerical analyses. Neglecting the elastic and inertial interactions between the train and the bridge, Lacarbonara and Colone [22] have developed a generalized numerical model, suitable to predict the dynamic response of tied-arch bridges, due to the passage of the three main European highspeed trains involving resonance phenomena. Yang et al. in [23] have analyzed the dynamic interaction between a short tied-arch bridge and moving loads, identifying the dynamic amplification factors for displacement and internal forces. However, most of the exiting studies on tied-arch bridges do not consider the influence of accurate discretization of the inertial forces, which typically are able to produce notable dynamic amplification effects on common design bridge variables $[9,14,15]$. Moreover, analyses available from the literature are focused on structures with relatively short spans, namely, up to $120 \mathrm{~m}$, and, to the Author's knowledge, no works on long-span bridges were properly developed in the literature. Therefore, the purpose of the present study is to analyze the dynamic behavior of tied-arch bridges in the field of medium and large span ranges, by evaluating the effects produced by the moving system on the bridge vibrations. It is worth noting that the present paper can be considered as an extension of previous authors' works $[9,14,15]$ in which the dynamic behavior was investigated in the framework of cable supported bridges. However, the main aims of this paper are to propose a parametric study in a dimensionless context in the framework of tied-arch bridges, which describes the relationship between dynamic amplification factors, moving loads, and bridge characteristics. The outline of the paper is as follows: in Section 2 a description of the tied-arch bridge together with the formulation of the bridge modeling and the evaluation of the initial configuration is presented. The numerical implementation is reported in Section 3, whereas in Section 4 numerical results are proposed.

\section{Theoretical Formulation: Initial Configuration and Equations of Motion}

Tied-arch bridge scheme, considered in the present paper, consists of two arch ribs whose extremities are rigidly connected to longitudinal edge beams which support the girder (Figure 1). Moreover, each arch rib carries tie beams by means of several vertical hangers spaced at constant step along the girder development. The whole system is simply supported at its ends. In particular, one end is pinned, whereas the other one is free to move longitudinally.

2.1. Initial Configuration of the Bridge. Since steel cables require posttensioning forces, the initial configuration of the bridge under the dead loading must be identified in advance. In order to calculate the initial stress in each cable, a numerical procedure developed, consistently with the zero displacement method being developed [24, 25]. In particular, the geometric initial shape of the bridge and the corresponding internal stresses of the cable system are obtained enforcing the girder to remain under dead loads in the undeformed design configuration. With reference to Figure 2, the unknown variables are represented by the posttensioning stresses in the cable:

$$
\underline{S}^{H}=\left(S_{1}^{H}, S_{2}^{H}, \ldots, S_{m}^{H}\right),
$$

where $m$ is the number of hangers of the cable arrangement. The displacement conditions utilized to achieve zero displacement variables at the girder anchorages are expressed as follows:

$$
\stackrel{\sim}{L}^{H}\left[\left(\bar{S}_{1}^{H}+\Delta S_{1}^{H}, \ldots, \bar{S}_{m}^{H}+\Delta S_{m}^{H}\right), \underline{U}_{3}^{H}\right]=0,
$$

where $\underline{\sim}^{H}$ is the constraint operator and $\underline{U}_{3}^{H}$ is the vector containing the vertical displacement of the cable at anchorage points located on the girder. It is worth nothing that in (2) the total initial stresses are expressed as a combination of a constant quantity $\left(\bar{S}_{i}^{H}\right)$ and an incremental contribution $\left(\Delta S_{i}^{H}\right)$. The former is a set of trial initial posttensioning cable forces, which are estimated by means of simple design rules commonly adopted in the context of bridge design [2628], whereas the latter is defined by an incremental value, representing the unknown quantity to be identified in the solving procedure.

2.2. Bridge Formulation and FE Implementation. The cable formulation is consistent with a large deformation theory based on the Green-Lagrange strain measure and the second Piola-Kirchhoff stress [29], whereas the material behavior is assumed to be linearly elastic. Nonlinearities in the cable elements are introduced to reproduce the local vibration effects, determined by the moving load application (Figure 3 ). The formulations of the arch and the girder are consistent with a geometric nonlinear model based on Euler-Bernoulli theory, in which large displacement is considered by using Green-Lagrange strain measure. Moreover, the torsional behavior owing to eccentric loading is described by means of the classical De Saint Venant theory. The external loads are consistent with uniformly distributed vertical moving forces and masses, travelling on the girder profile at constant speed $(c)$ and at a fixed eccentricity $(e)$ with respect to the geometrical axis of the girder. In particular, the definition of the moving loads is consistent with previous author's works, in which a refined description of the moving loads in terms 


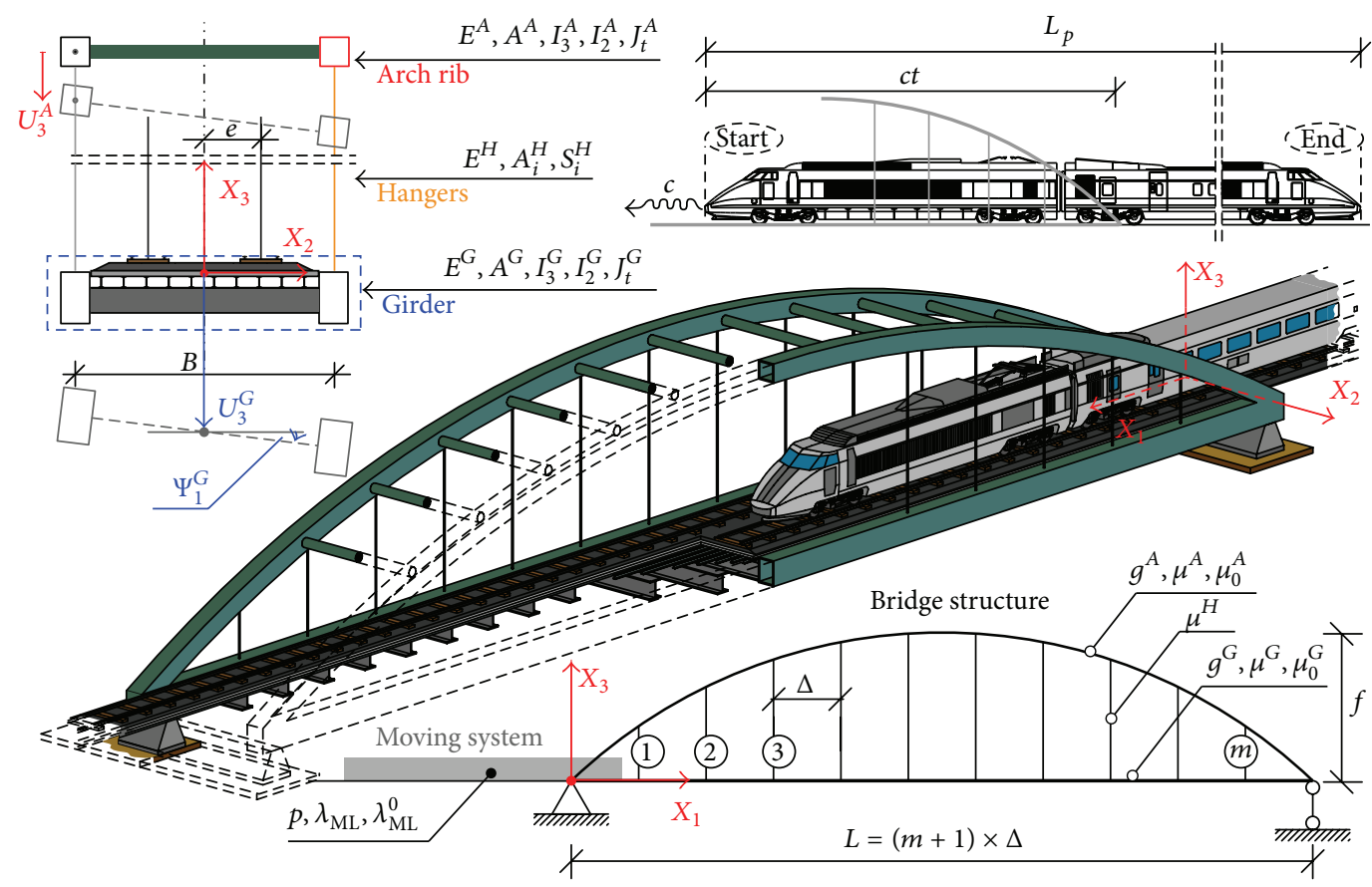

FIGURE 1: Structural scheme of the tied-arch bridge.

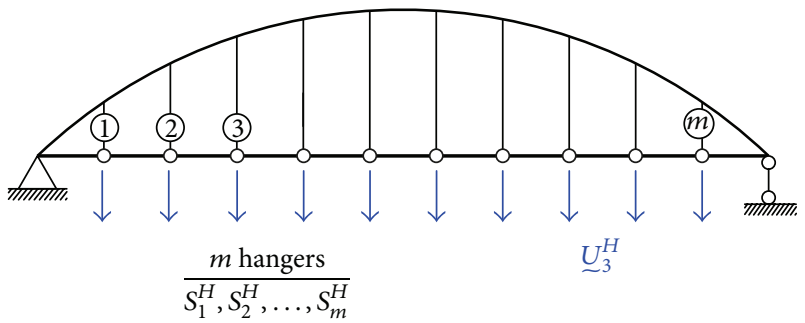

Figure 2: Displacement and control variables for the evaluation of the initial configuration.

of nonstandard terms in the acceleration functions as well as the time dependent effects on the mass distribution is considered. More details on the analytical expressions can be recovered in $[14,15]$ or in the appendix, in which essential equations concerning moving load description are briefly reported. Finite element expressions are written, introducing Hermit cubic interpolation functions for the girder and arch flexures and Lagrange linear interpolation functions for the cable system, girder, and arch displacement:

$$
\begin{aligned}
& \underline{U}^{H}(\underline{\sim}, t)=\underline{N}^{H}(\underline{r}) \underline{\sim}^{q^{H}}(t), \\
& \underline{\sim}^{G}(\underset{\sim}{r}, t)=\underline{N}^{G} \underline{\sim}^{G}(t), \\
& \underline{U}^{A}(\underline{r}, t)=\underline{N}^{A} \underline{\sim}^{A}(t) \text {, }
\end{aligned}
$$

where $q^{H}, q^{G}$, and $q^{A}$ are the vectors collecting the nodal degrees of freedom of the hanger cable, girder, and arch, respectively, $\mathbb{N}^{H}, \mathbb{N}^{G}$, and $\underline{N}^{A}$ are the matrixes containing the displacement interpolation function for hanger, girder, and arch, and $\underset{\sim}{r}$ is the local coordinate vector of the generic finite element. The discrete equations in the local reference system of the $i$ th element lead to the following equations in matrix notation:

$$
\begin{aligned}
& \left(\underline{M}_{S}^{G}+\underline{M}_{N S}^{G}\right) \underline{\tilde{U}}^{G}+\left(\underline{C}_{S}^{G}+\underline{C}_{N S}^{G}\right) \underline{\dot{U}}^{G} \\
& +\left(\underline{K}_{S}^{G}+\underline{K}_{N S}^{G}\right) \underline{U}^{G}=\underline{\sim}^{G}+\underline{Q}^{G}, \\
& \underline{M}^{A} \underline{\tilde{U}}^{A}+\underline{C}^{A} \underline{\sim}^{A}+\underline{K}^{A} \underline{U}^{A}=\underline{\sim}^{A}+\underline{Q}^{A}, \\
& \underline{M}^{H} \ddot{U}^{H}+\underline{C}^{H} \underline{\tilde{U}}^{H}+\underline{K}^{H} \underline{U}^{H}=\underline{P}^{H}+\underline{Q}^{H},
\end{aligned}
$$

where $(\underline{M}, \underline{C}, \underline{K})$ are standard $(S)$ mass, damping, and stiffness matrixes, $\underset{\sim}{P}$ is the load vector produced by the dead and live loading, and $Q$ is the unknown force vector collecting the point sources. Moreover, the matrixes concerning nonstandard terms denoted by the subscripts $(\cdot)_{N S}$ and introduced essentially by the presence of the moving loads are reported in explicit form in the Appendix. In order to reproduce the bridge kinematic correctly, additional relationships to define the connections between girder, arch, and hangers are necessary. In particular, hanger displacement should be equal to that of the girder and the arch at the corresponding intersection points; thus, the bridge kinematic is restricted by means of the following constrain equations:

$$
\begin{aligned}
U_{3}^{G}\left(\underline{\sim}_{i}^{G}, t\right)+\Psi_{1}^{G}\left(\underline{\sim}_{i}^{G}, t\right) \frac{B}{2} & =U_{3}^{H}\left(\underline{X}_{i}^{H}, t\right), \\
U_{1}^{G}\left(\underline{\sim}_{i}^{G}, t\right)-\Psi_{3}^{G}\left(\underline{\sim}_{i}^{G}, t\right) \frac{B}{2} & =U_{1}^{H}\left(\underline{X}_{i}^{H}, t\right), \\
U_{1}^{A}\left(\underline{\sim}_{A}, t\right) & =U_{1}^{H}\left(\underline{X}_{H}, t\right), \\
U_{2}^{A}\left(\underline{\sim}_{A}, t\right) & =U_{2}^{H}\left(\underline{\sim}_{H}, t\right), \\
U_{3}^{A}\left(\underline{\sim}_{A}, t\right) & =U_{3}^{H}\left(\underline{X}_{H}, t\right),
\end{aligned}
$$




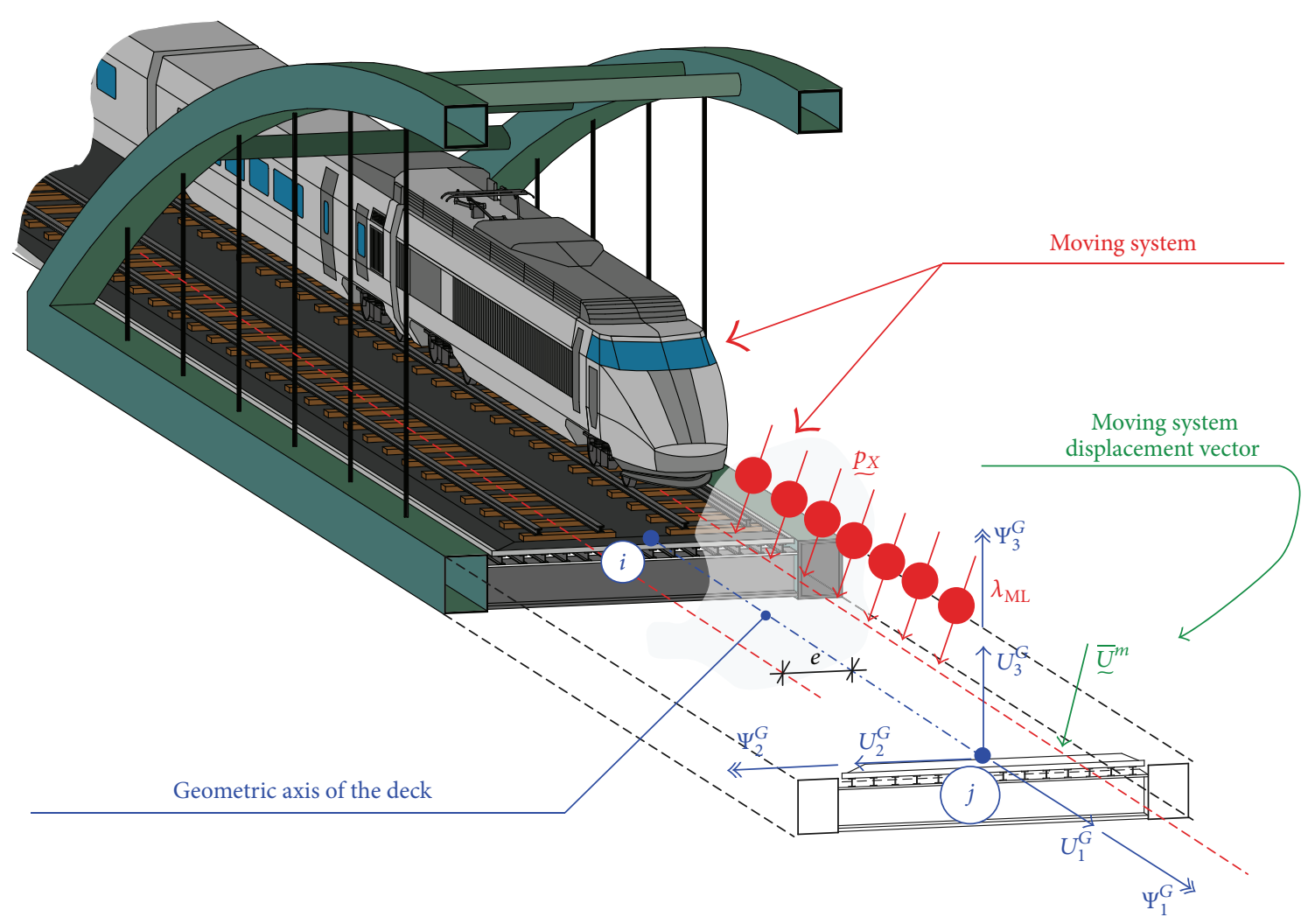

FIGURE 3: Moving load description and girder kinematic.

where ${\underset{\sim}{X}}_{i}^{H},{\underset{\sim}{i}}_{i}^{G}$, and ${\underset{\sim}{i}}_{i}^{A}$ represent the vectors containing the intersection positions at the $i$ th anchorage point of the hanger, girder, and arch, respectively. Finally, starting from (4)-(6), taking into account (7) as well as the balance of secondary variables at the interelement boundaries, the resulting equations of the finite element model are:

$$
\underline{M} \underline{Q}+\underline{C} \dot{Q}+\underline{K} \underline{Q}=\underset{\sim}{R}
$$

where $Q$ with $Q=\underline{U}_{H} \cup \underline{U}_{D} \cup \underline{U}_{A}$ is the generalized coordinate vector containing the kinematic variables associated with the

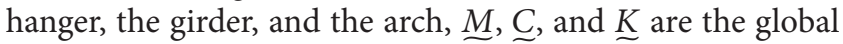
mass, stiffness, and damping matrixes, and $\underset{\sim}{R}$ is the loading vector. Since the structural behavior of each element depends on the deformation state of the members, the governing equations defined by (8) will change continuously as the structure deforms. Moreover, the external loads owing to the presence of its own moving mass determine a time dependent mass distribution function on the girder profile. Consequently, the discrete equations are affected by nonlinearities in the stiffness matrix and time dependence in the mass matrix. The governing equations are solved numerically, using a user customized finite element program, that is, COMSOL Multiphysics $^{\mathrm{TN}}$ version 4.4 [30]. The analysis is performed by means of two different stages. Initially, a preliminary analysis is devoted to calculating the initial stress distribution in the cable system, that is, "zero configurations." In this context, the shape optimization procedure is developed, consistently with a Newton-Raphson iteration scheme. Since the loading condition refers to the application of dead loading only, the analysis is developed in the framework of a static analysis. The algebraic equations concerning the moving load problem are solved by a direct integration method, which is based on an implicit time integration scheme. In particular the generalized- $\alpha$ method, which is an implicit, second-order accurate algorithm, was employed and the value of alpha was fixed to 0.25 . Moreover, a Newton-Raphson scheme in the time step increment based on the secant formulation is utilized for the nonlinearities involved in the governing equations [30].

\section{Results}

A parametric study is proposed, with purpose to identify the DAFs of typical bridge design variables. In particular, the investigation is performed in terms of dimensionless variables, strictly related to both moving loads and bridge characteristics. In order to quantify the amplification effects produced by the moving loads over the static solution (ST), numerical results are presented in terms of dynamic amplification factors, defined as follows:

$$
\Phi^{X}=\frac{\max (X, t=0, \ldots, T)}{X_{\mathrm{ST}}}
$$


where $X$ is the generic variable under investigation, $T$ is the observation period, and the subscript $(\cdot)_{\mathrm{ST}}$ refers to the static value of the variable. The bridge and moving load characteristics are selected consistently with values utilized in practical applications [31], which are in agreement with common code prescriptions on bridge engineering [32,33]. The following parameters related to aspect ratio, stiffness, cable system properties, and dead and live loads involved in the structure are adopted:

$$
\begin{aligned}
\frac{f}{L} & =\frac{1}{5} ; \\
\frac{B}{L} & =\frac{1}{15} ; \\
\varepsilon_{F} & =\frac{I_{2}^{A}}{I_{2}^{G}}=[0.05-0.2] ; \\
\varepsilon_{A} & =\frac{A^{A}}{A^{G}}=[0.5-2] ; \\
\frac{S_{a}^{H}}{E^{H}} & =0.002 ; \\
m & =[11,15,19,23] ; \\
\Delta & =\frac{L}{(m+1)}, \\
g_{\text {tie }}^{G}+g^{A} & =\frac{R\left(g_{\text {nnstr }}^{G}+1.1 p\right)}{1-R} \\
\text { with } & R=0.088+0.00321 \cdot L(f t) ; \frac{g_{\text {nnstr }}^{G}}{p}=0.95,
\end{aligned}
$$

where $L$ is the bridge length, $f$ is the arch rise, $B$ is the girder width, $\varepsilon_{F}$ and $\varepsilon_{A}$ are the relative bending and axial stiffness, $E^{H}$ is the cable elasticity modulus, $S_{a}^{H}$ is the cable allowable stress, and $m$ is the number of hangers which are uniformly distributed along the girder at constant step $\Delta$.

Moreover, $g_{\text {tie }}^{G}$ and $g_{\text {nnstr }}^{G}$ represent the loads per unit length of tie and utilities of the girder, respectively, whereas $g^{A}$ is the load per unit length of the arch. It is worth nothing that the parameter $R$ defines the dead load ratio between the weight involved in both girder and arch and the one of the whole structure; such value is assumed to be consistent with comparative studies developed in the framework of tied-arch bridges [31]. The entity of the moving system $(p)$ is consistent with LM-71 train model, whose characteristics are reported in [32]. The cross section area of the $i$ th hanger, namely, $A_{i}^{H}$, is designed in such a way that the dead loads of the girder $\left(g^{G}=g_{\text {tie }}^{G}+g_{\text {nnstr }}^{G}\right)$ produce constant stresses over all the distributed elements, which are assumed to be equal to a fixed design value, namely, $S_{g}^{H}$. Such design stress is defined on the basis of the ratio between live $(p)$ and self-weight loads $\left(g^{G}\right)$ and allowable cable stress $\left(S_{a}^{H}\right)$ by mean of the following relationship:

$$
S_{g}^{H}=\frac{g^{G}}{g^{G}+p} S_{a}^{H} .
$$

Therefore, the geometric measurement for the cables system can be expressed by the following expression $[26,27]$ :

$$
A^{H}=\frac{g^{G} \Delta}{S_{g}^{H}} .
$$

At first, the behavior of the bridge is analyzed to investigate the relationship between dynamic amplification factors (DAFs) and normalized speed of the moving system, which is defined by means of the following expression:

$$
\vartheta=c\left(\frac{S_{a}^{H} \mu^{G}}{E^{H} g^{G} B}\right)^{1 / 2},
$$

where $\mu^{G}$ is the mass per unit length of the girder and $E^{H}$ is the elasticity modulus of hangers. In particular, in order to point out the influence of the moving mass on the bridge dynamic behavior, analyses are developed by using the following three different models for the inertial description:

(a) External mass of the moving system completely neglected, known as Moving Force Model (MFM).

(b) Inertial description of the moving system neglected with respect to nonstandard inertial forces (Coriolis acceleration and centripetal acceleration in (A.5) are not considered), namely, Standard Analysis (SA).

(c) Inertial description of the moving system according to (A.5), namely, Nonstandard Analysis (NSA).

The investigated structure is consistent with a steel tiedarch bridge of $300 \mathrm{~m}(L)$ whose values of relative stiffness, number of hangers, and live-to-dead load ratio are assumed to be equal to $\varepsilon_{F}=0.1, \varepsilon_{A}=1, m=15$, and $p / g^{G}=$ 0.67 , respectively. At first, natural frequencies and the mode shapes of the bridge are obtained by means of prestressed modal analysis, in which the initial configuration concerning the distribution of stresses and deformations is considered. Such analysis, whose results for the six mode shapes are reported in Figure 4, is developed to determine a suitable time step size for the transient analysis and to investigate resonance phenomena due to the application of the external loads. In particular, since a reasonable approximation of the dynamic solution may be obtained involving the first six mode shapes of the arch bridge, the time integration step is assumed to be equal to $1 / 20$ of the period associated with the sixth mode of vibration. Moreover, Rayleigh damping model is used; that is, $\underline{C}=a \underline{M}+b \underline{K}$, where $a$ and $b$ are two constants of the damping model, whose values, equal to $0.1393 \mathrm{~L} / \mathrm{s}$ and $0.0155 \mathrm{~s}$, respectively, are determined on the basis of two vertical modes and taking a modal damping ratio equal to $v=0.05$. The moving system is assumed to proceed at constant speed $c$ from left to right along the bridge development. In Figure 5 results concerning the DAFs for vertical displacement $\left(\Phi^{U_{3}^{G}}\right)$ at $1 / 2$ and $3 / 4$ girder crosssections, that is, $X_{1} / L=0.50$ and $X_{1} / L=0.75$, respectively, are presented. The DAF evolution curves denote a tendency to increase with the speeds of the moving system. In particular, the DAFs at $X_{1} / L=0.75$ are generally larger than the 


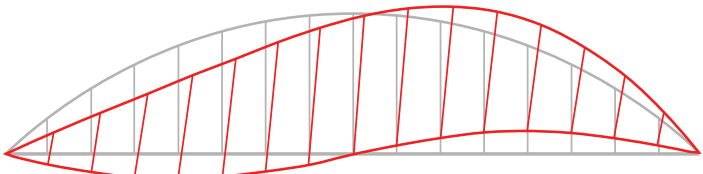

Freq. $=0.3249 \mathrm{~Hz}$

(a)

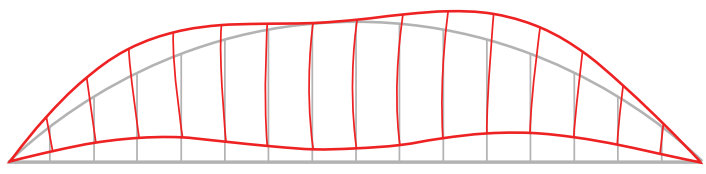

Freq. $=1.1407 \mathrm{~Hz}$

(c)

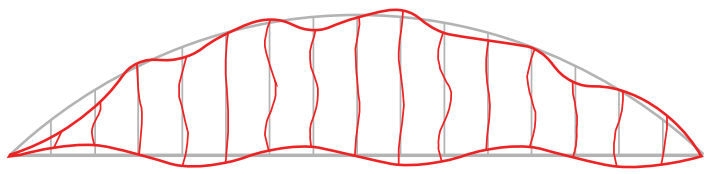

Freq. $=5.0403 \mathrm{~Hz}$

(e)

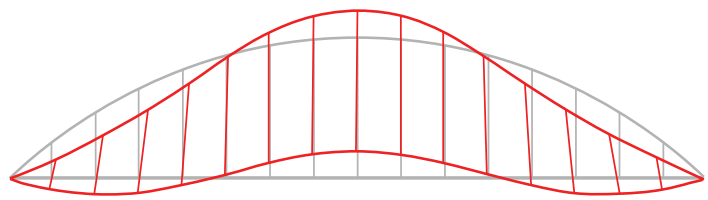

Freq. $=0.6981 \mathrm{~Hz}$

(b)

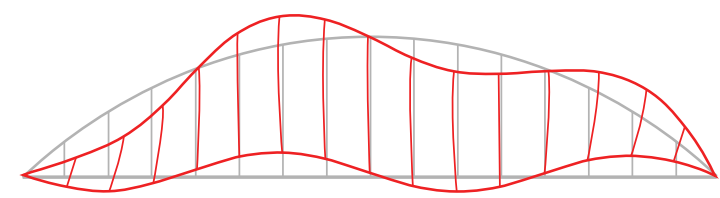

Freq. $=1.2791 \mathrm{~Hz}$

(d)

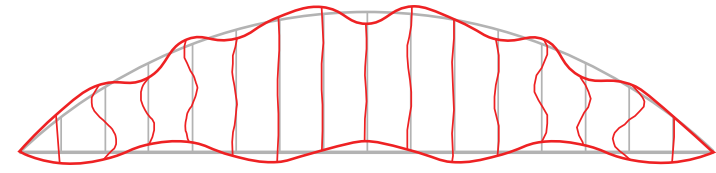

Freq. $=6.0962 \mathrm{~Hz}$

(f)

FIGURE 4: Representative mode shapes of the bridge.

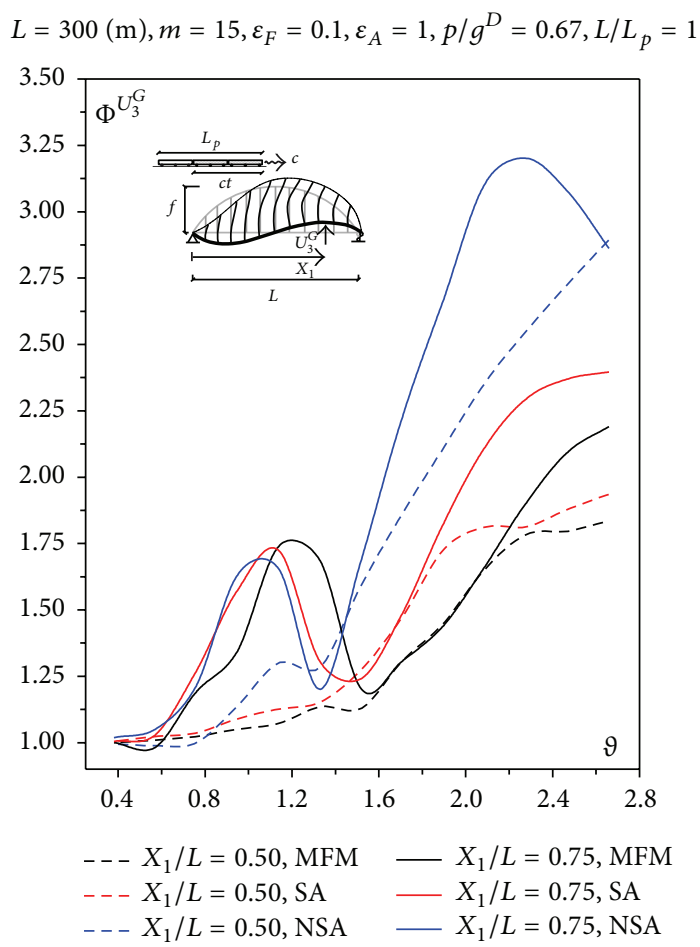

FIGURE 5: Dynamic amplification factors for girder vertical displacement $\left(\Phi^{U_{3}^{G}}\right)$ versus normalized speed parameter ( 9$)$. 
corresponding ones observed for the midspan cross section for each value of the normalized speed. The mass of the moving system affects the dynamic behavior of the bridge mainly at high range of speed, that is, for $1.60 \leq \vartheta \leq$ 2.60 , where nonstandard terms in the acceleration function provide the largest amplification. In this context, considerable underestimations in the DAF predictions are noted if the travelling mass is not properly evaluated. In particular, for the midspan cross section, the increments of the maximum value of the DAFs are from 1.12 to 1.83 and from 1.27 to 1.93 for MFM and SA formulation, respectively, and from 1.53 to 2.89 for the NSA formulation. Similarly, at $X_{1} / L=$ 0.75 , the DAFs increase in the ranges of [1.20-2.18], [1.242.39], and [1.69-2.86] for MFM, SA, and NSA formulations, respectively. Contrarily, for reduced values of moving system speeds, results arising from dynamic and static solutions are practically coincident and, consequently, the influence of the external mass becomes negligible. It is worth nothing that the bridge is affected by notable dynamic amplification also for medium speeds, since large peaks of values in the DAF curves, that is, close to 1.75 , are observed, especially for the analysis at $X_{1} / L=3 / 4$. This behavior can be explained by resonance issues, which occur when the moving system excitation frequencies are close to ones involved in the bridge structure. In order to verify such aspect and the differences in the dynamic behavior of the bridge for various moving system speeds, vertical acceleration at $X_{1} / L=3 / 4$ is analyzed in the frequency domain, by means of the Fast Fourier Transform (FFT) analysis. In particular, comparisons in terms of mass description, that is, between NSA and MFM, are proposed by means of time histories of displacement and acceleration functions and force magnitude of the FFT (Figure 6):

(i) Dynamic response of the bridge for a relatively low speed of the moving system, that is, $\vartheta=0.76$ (Figure 6(a)).

(ii) Results for normalized speed values equal to $\vartheta=$ 1.102 and $\vartheta=1.235$, which represent the resonance speeds in the case of NSA and MFM formulations (Figure 6(b)).

(iii) Dynamic response for moving system speeds which involve the maximum DAFs for vertical displacement of the girder in the case of NSA and MFM at $\vartheta=2.28$ and $\vartheta=2.66$ (Figure 6(c)).

From the results, it transpires that both NSA and MFM lead to comparable predictions in terms of vertical displacement and acceleration for low values of speeds since the effects of the external mass of the moving system are quite reduced. The FFT analysis denotes that, in the range of the investigated moving system speed, the dynamic response of the bridge is dominated mainly by its fundamental mode (Figure 4(a)), since peaks of values in the FFT diagram at a value of frequency close to the fundamental one of the bridge, namely $0.325 \mathrm{~Hz}$, are observed. However, for high-speed values, that is, $\vartheta=2.28$ or $\vartheta=2.66$, the results show that contributions arising from high frequencies start to affect the dynamic behavior of the structure. Such influence is much marked for the analysis developed in the case of NSA model, in which a refined description of the inertial forces including nonstandard acceleration terms in the inertial forces is considered. This aspect can be well appreciated by means of the FFT diagram reported in Figure 6(c), which presents several peaks of values close to the natural frequencies of the bridge, that is, $0.325 \mathrm{~Hz}, 0.701 \mathrm{~Hz}, 1.012 \mathrm{~Hz}$, and $1.207 \mathrm{~Hz}$ (Figures 4(a), 4(b), and 4(c)). Moreover, in the range of low or medium speeds, NSA and MFM formulations lead basically to the same predictions, since the contributions of nonstandard acceleration become quite negligible. The influence of the external mass on the internal stress resultants variables of the bridge in terms of moving mass description is also examined in Figures 7 and 8, in which the distributions of DAFs for bending moment and axial force in the girder and arch profiles, respectively, for increasing values of normalized speeds are depicted. For the sake of brevity, only results at high speeds of the moving system are reported, in which typically the major dynamic amplification is observed. Similar results are presented in Figure 9, in which the distributions of the DAFs for the maximum axial force in hangers are illustrated. The results indicate that at high speeds relevant DAFs are observed with values much larger than those determined for the kinematic quantities in the arch and girder. Moreover, underestimations in the DAFs prediction are noted, if nonstandard inertial forces arising from Coriolis acceleration and centripetal acceleration are neglected. In particular, for the girder or the arch, the maximum values of DAFs for bending moment achieved by NSA are larger than those predicted by other moving system models and the corresponding percentage errors are between [28.69/27.5] or [24.21/22.88] for the MFM and SA, respectively. Moreover, the differences between the NSA and other moving system models in terms of the maximum DAFs of the axial force are equal to $11.22 \%$ and $8.42 \%$, for the girder, and $21.33 \%$ and $16.66 \%$, for the arch, respectively. For the hangers, the maximum value of DAFs obtained by the NSA differs with errors equal to $6.75 \%$ and $8.10 \%$ for MFM and SA formulations, respectively. Moreover, the maximum DAF observed for hangers is equal to 1.49 , which is smaller than the failure stress typically observed in cable elements; that is, $S / S_{a}=2.25$. It is worth nothing that the DAFs of the bending moment in the girder are much larger than those observed for the arch with percentage errors equal to $44 \%, 45 \%$, and $47 \%$ for MFM, SA, and NSA, respectively.

Additional analyses, reported in Figures 10(a) and 10(b), are developed with the purpose to investigate the influence of the loading strip length ratio $\left(L_{p} / L\right)$ and the normalized speed $(\vartheta)$ of the moving system on the DAFs of the vertical displacement at $X_{1} / L=0.75$ and $X_{1} / L=0.5$ girder cross sections. For the sake of brevity, only results achieved by NSA model are presented. However, for completeness, the percentage errors in terms of the DAFs between NSA and other moving system models are reported in Tables 1 and 2. From the analyses, it transpires that the dynamic bridge behavior appears to be quite dependent from the loading strip length. As a matter of fact, results at $X_{1} / L=0.75$ girder cross section reported in Figure 10(a) show that the dynamic amplification generally grows with the loading the strip length and strongly depends on the moving system 


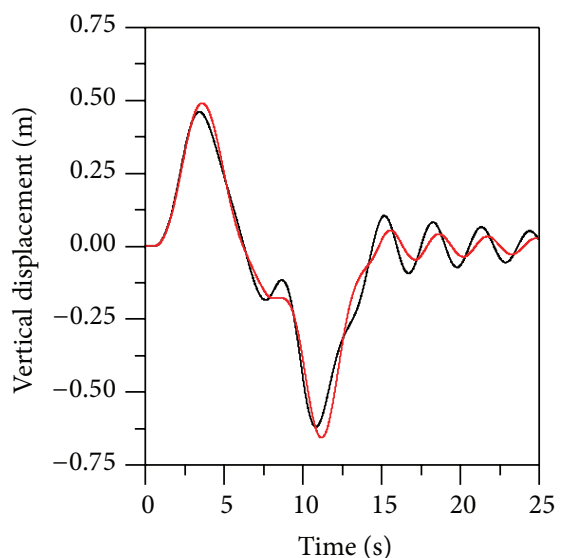

- MFM, $\vartheta=0.76$

NSA, $\vartheta=0.76$
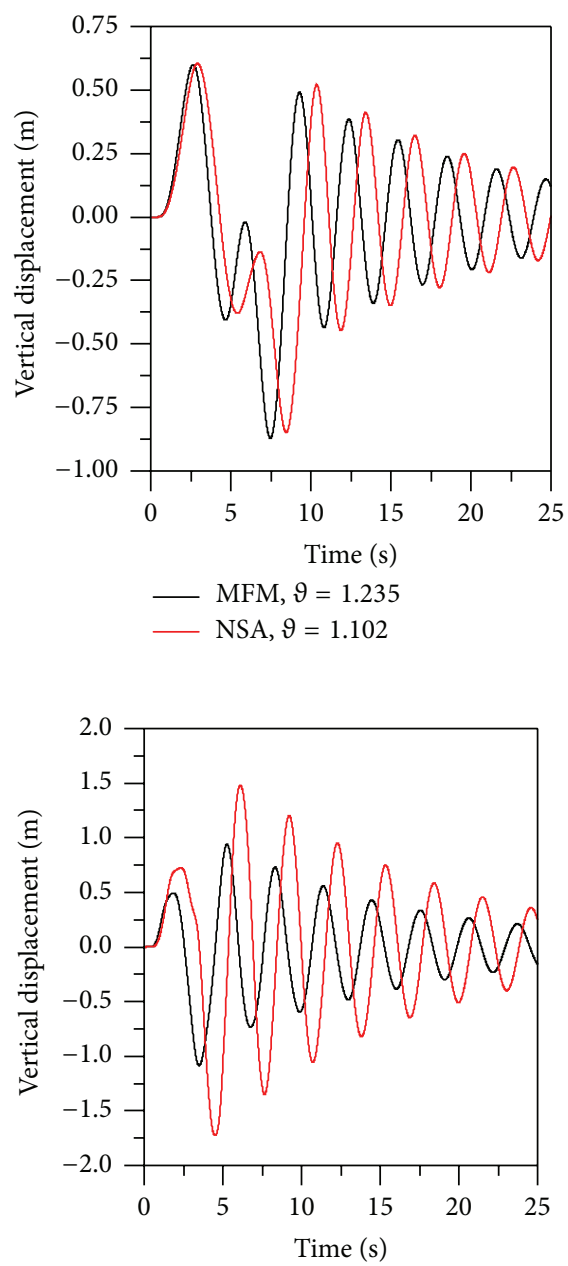

— MFM, $\vartheta=2.66$

NSA, $9=2.28$

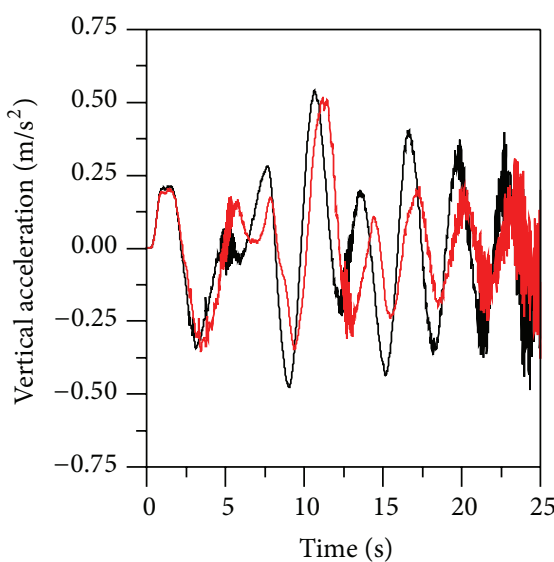

MFM, $\vartheta=0.76$

NSA, $\vartheta=0.76$

(a)

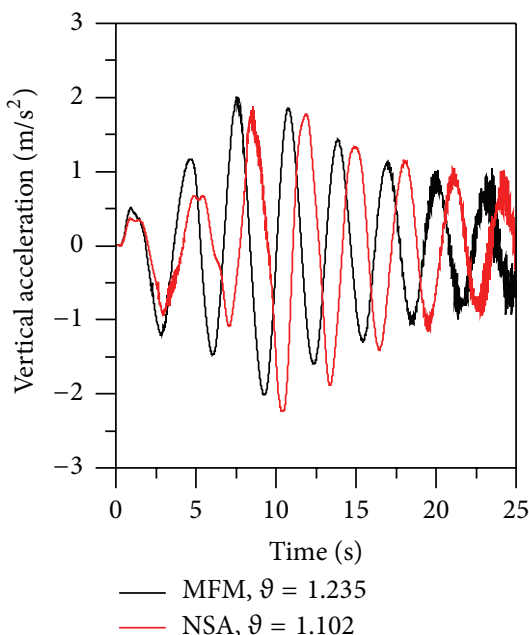

(b)

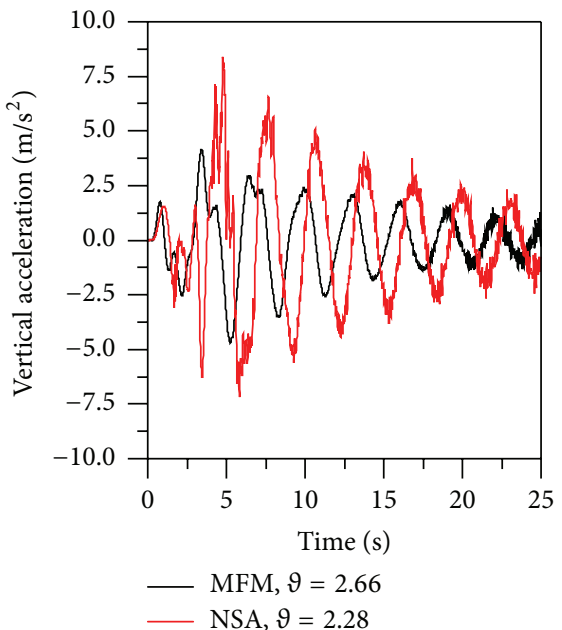

(c)

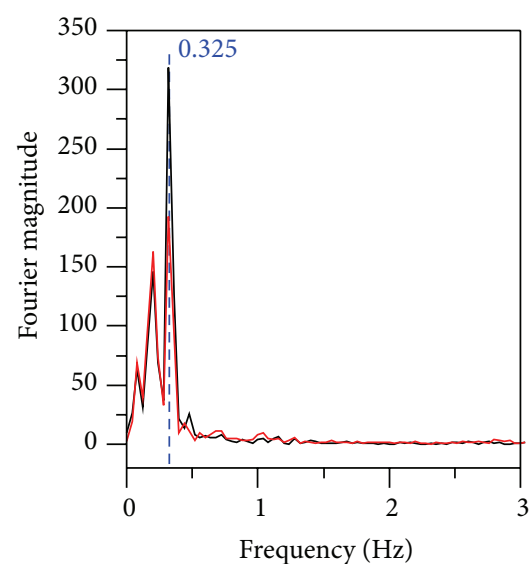

MFM, $\vartheta=0.76$

NSA, $\vartheta=0.76$
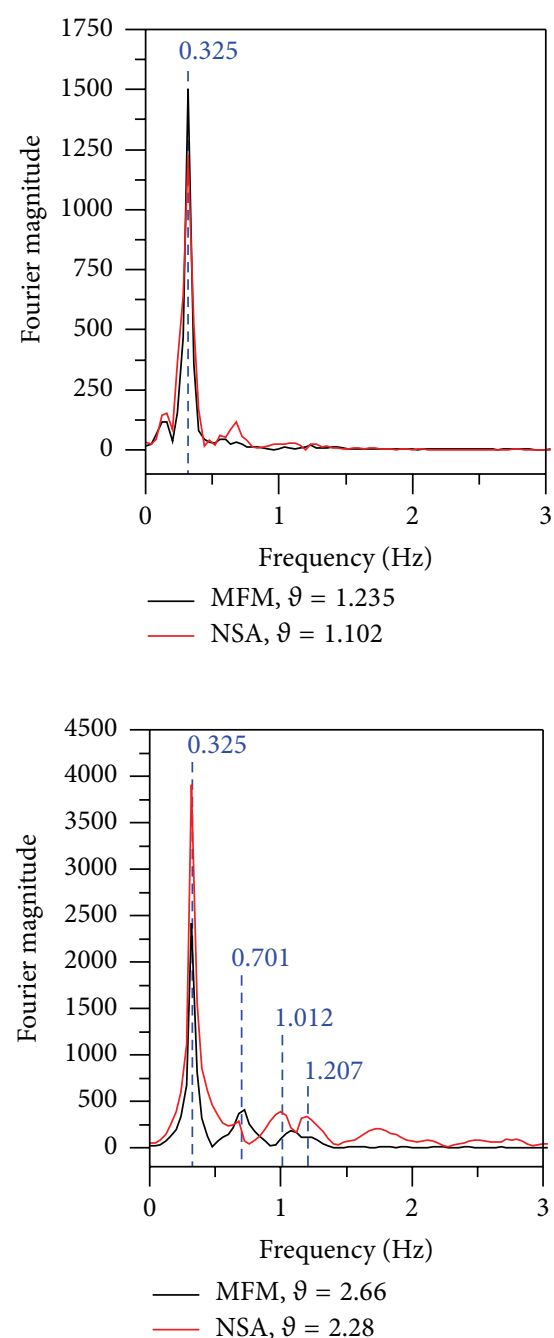

FIGURE 6: Time histories of vertical displacement and acceleration for girder cross section at $X_{1} / L=0.75$ and analysis in frequency domains of vertical acceleration. 


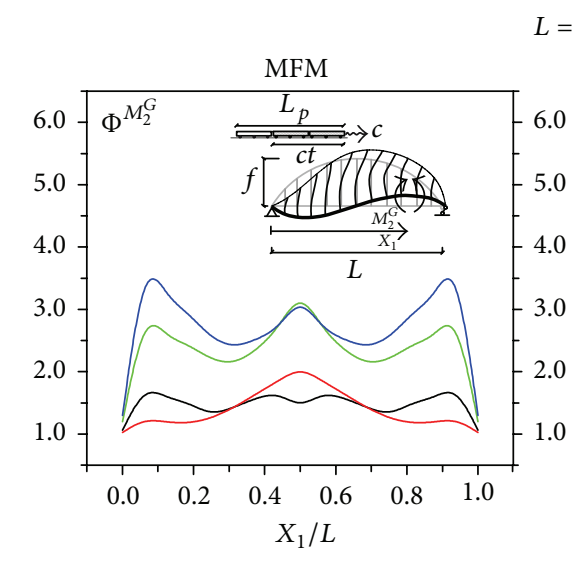

$L=300(\mathrm{~m}), m=15, \varepsilon_{F}=0.1, \varepsilon_{A}=1, p / g^{G}=0.67, L / L_{p}=1$
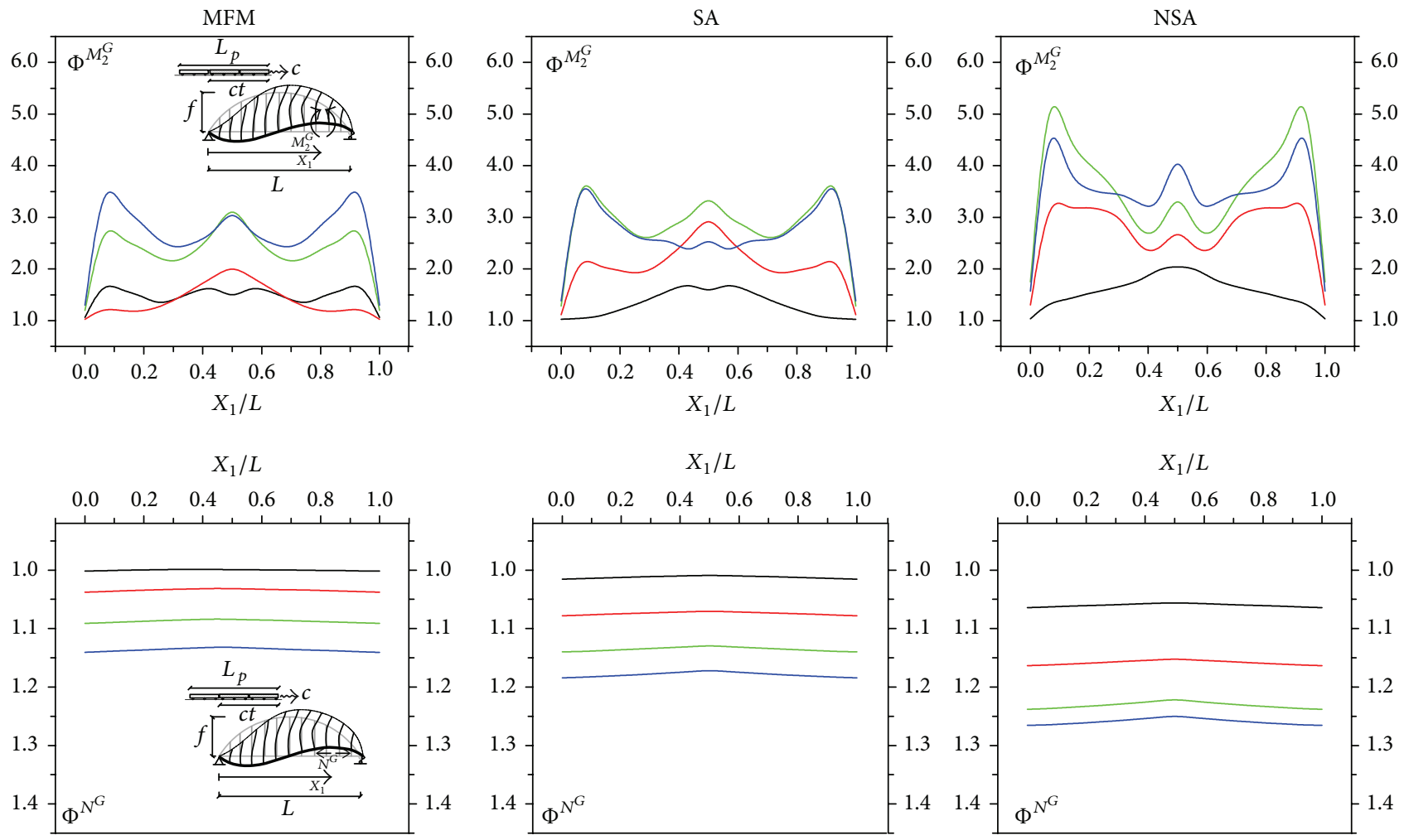

$X_{1} / L$

$$
X_{1} / L
$$

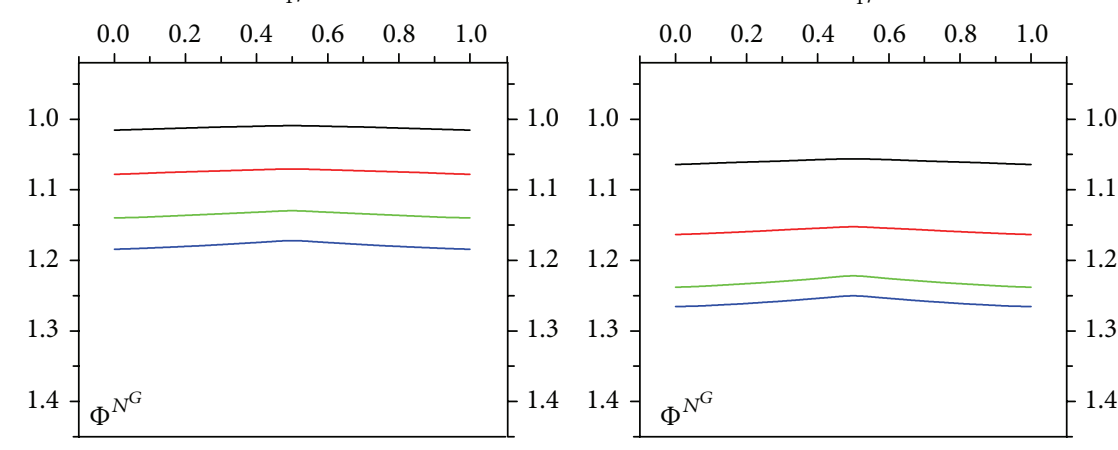

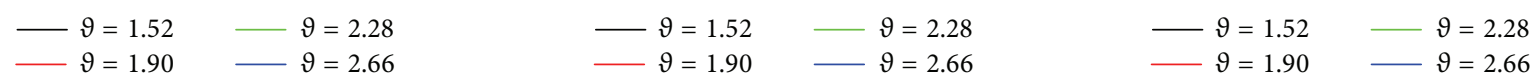

FIGURE 7: DAFs curves for bending moment and axial force in the girder.
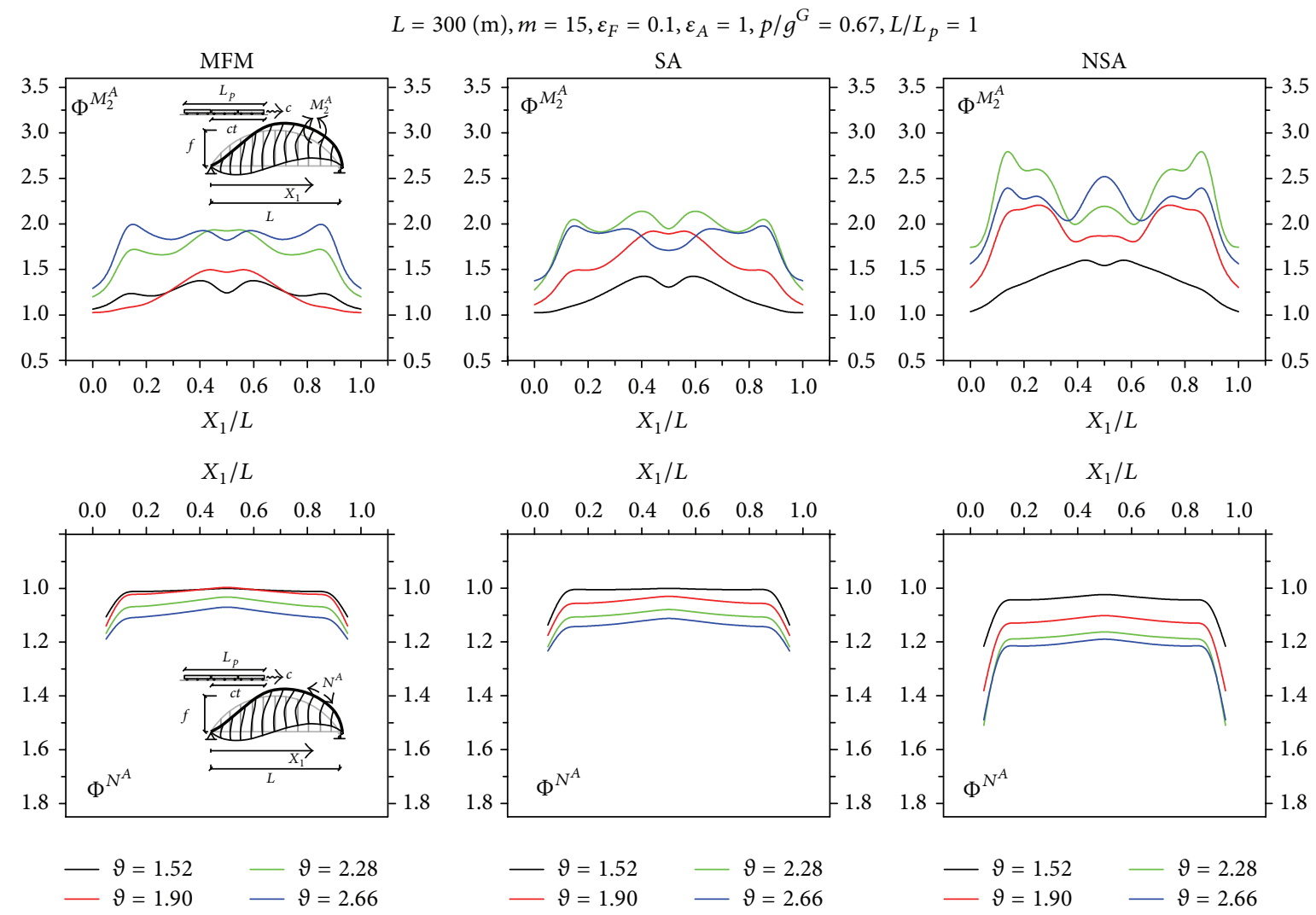

FIGURE 8: DAFs curves for bending moment and axial force in the arch. 
$L=300(\mathrm{~m}), m=15, \varepsilon_{F}=0.1, \varepsilon_{A}=1, p / g^{G}=0.67, L / L_{p}=1$
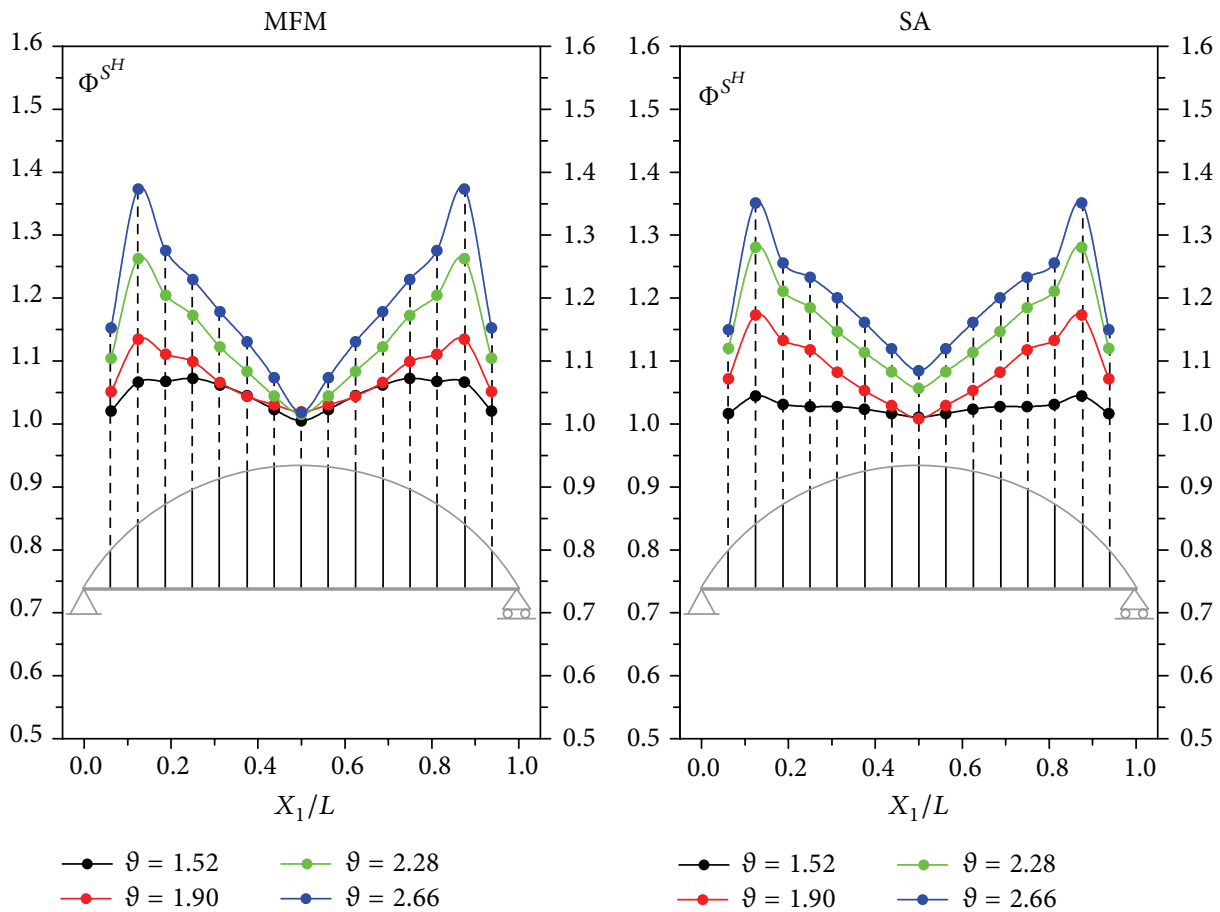

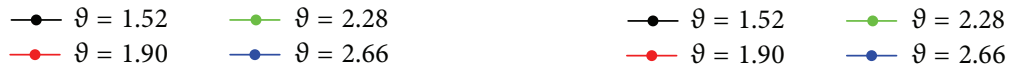

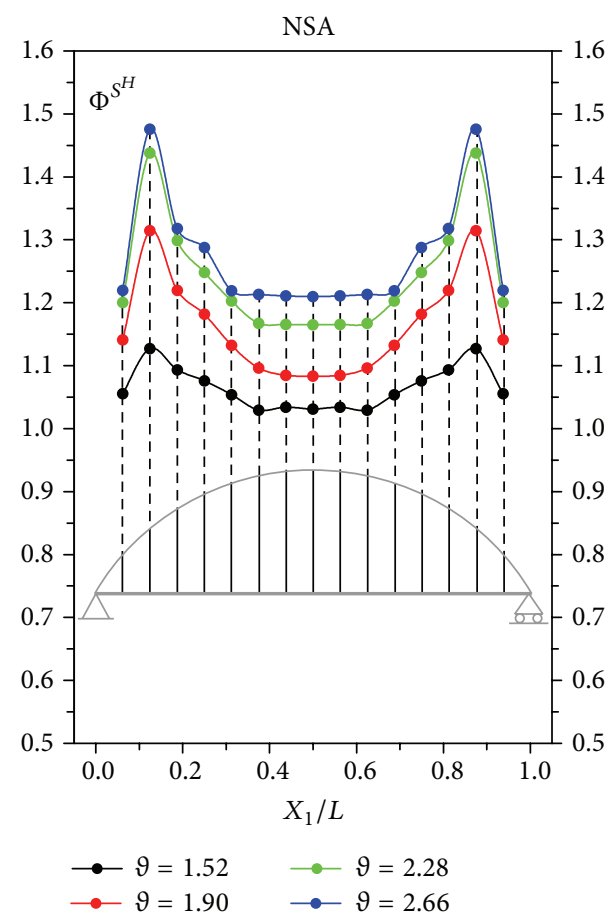

FIGURE 9: DAFs curves for the maximum axial force in hangers.

speeds. In particular, for short loading strip length, the bridge achieves the maximum amplification in the medium range of speeds, that is, for $1.5 \leq \vartheta \leq 1.7$. Contrarily, for large loading strip lengths, the inertial forces of moving system greatly affect the dynamic behavior of the bridge, especially with the contributions arising from Coriolis acceleration and centripetal acceleration (Table 1); as a consequence, the maximum amplification is observed in the high range of speeds. On the other hand, results concerning DAFs at the midspan cross section (Figure 10(b)) are quite unaffected 


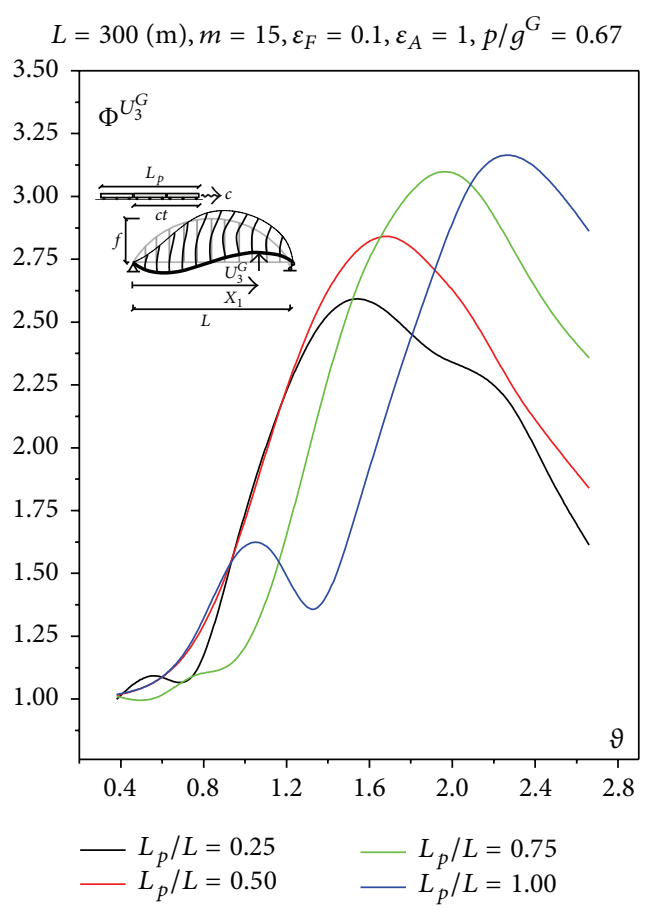

(a)

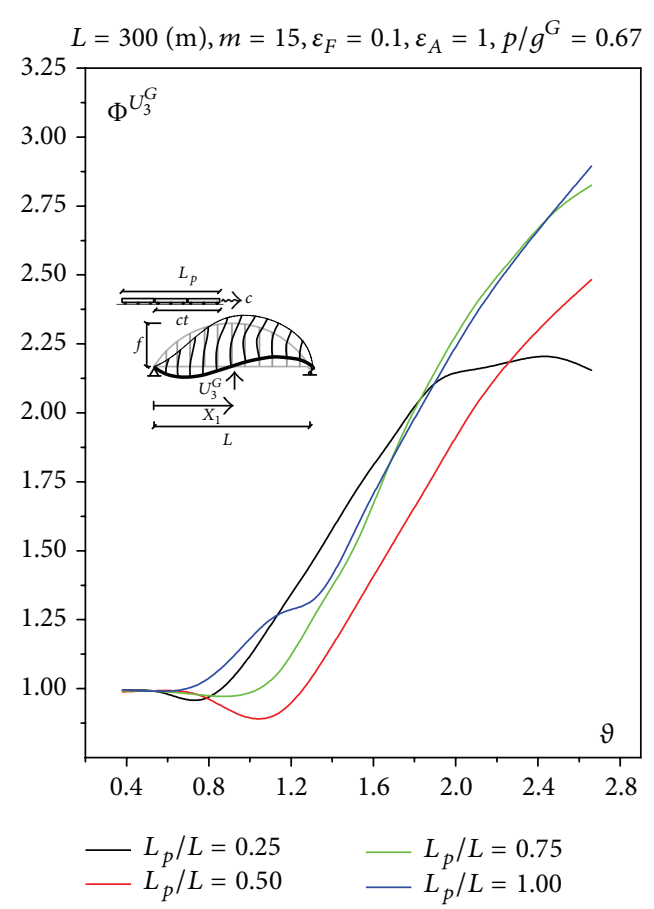

(b)

FIGURE 10: Influence of moving system length $\left[L_{p}\right]$ : DAFs for vertical displacement $\left[\Phi^{U_{3}^{G}}\right]$ versus normalized speed parameter [9] at 3/4 (a) and $1 / 2$ (b) girder cross sections.

TABLe 1: Percentage errors of vertical displacement dynamic amplification factors $\left(\Phi^{U_{3}^{G}}\right)$ at $X_{1} / L=0.75$ between the Moving Force Model $(\mathrm{MFM})$, Standard Analysis (SA), and Nonstandard Analysis (NSA) for different normalized speed parameters $(\mathcal{\vartheta})$.

\begin{tabular}{|c|c|c|c|c|c|c|c|c|}
\hline \multirow[t]{2}{*}{$\vartheta$} & \multicolumn{2}{|c|}{$\begin{array}{c}L_{p} / L=0.25 \\
\text { Error }(\%)\end{array}$} & \multicolumn{2}{|c|}{$\begin{array}{c}L_{p} / L=0.50 \\
\text { Error }(\%)\end{array}$} & \multicolumn{2}{|c|}{$\begin{array}{c}L_{p} / L=0.75 \\
\text { Error }(\%)\end{array}$} & \multicolumn{2}{|c|}{$\begin{array}{c}L_{p} / L=1.00 \\
\text { Error }(\%)\end{array}$} \\
\hline & MFM & SA & MFM & SA & MFM & SA & MFM & SA \\
\hline 0.38 & 0.08 & 0.11 & 1.50 & 0.08 & 1.12 & 1.44 & 1.82 & 1.47 \\
\hline 0.57 & 4.82 & 2.47 & 5.21 & 0.44 & 1.79 & 0.78 & 6.43 & 1.99 \\
\hline 0.76 & 1.27 & 1.28 & 2.32 & 0.19 & 7.50 & 1.03 & 1.89 & 4.49 \\
\hline 0.95 & 7.31 & 2.97 & 16.23 & 4.73 & 8.54 & 1.24 & 17.54 & 3.52 \\
\hline 1.14 & 7.26 & 2.01 & 18.12 & 6.75 & 35.21 & 20.94 & 5.21 & 4.16 \\
\hline 1.33 & 7.37 & 4.75 & 16.50 & 8.90 & 35.57 & 18.39 & 40.37 & 8.62 \\
\hline 1.52 & 7.60 & 9.27 & 14.49 & 9.98 & 35.98 & 20.63 & 29.01 & 26.61 \\
\hline 1.71 & 5.22 & 3.69 & 12.45 & 9.87 & 29.35 & 17.85 & 41.33 & 33.23 \\
\hline 1.9 & 2.19 & 1.74 & 7.15 & 6.48 & 25.34 & 16.98 & 46.05 & 31.59 \\
\hline 2.09 & 6.42 & 2.19 & 3.19 & 4.00 & 20.09 & 14.74 & 46.44 & 31.97 \\
\hline 2.28 & 8.66 & 9.28 & 5.09 & 3.47 & 10.72 & 6.23 & 40.53 & 28.28 \\
\hline 2.47 & 0.33 & 0.49 & 9.48 & 6.74 & 1.29 & 1.45 & 31.88 & 22.93 \\
\hline 2.66 & 7.32 & 8.01 & 14.04 & 13.05 & 2.68 & 3.43 & 23.50 & 16.28 \\
\hline
\end{tabular}

by the loading strip length, since comparable predictions are obtained from each of the investigated moving system lengths.

The dynamic behavior of the bridge is investigated with respect to the relative dimensionless arch/girder stiffness ratio $\left(\varepsilon_{F}\right)$. Such quantity is able to identify the interaction between arch and girder in terms of relative and global stiffness of the bridge. For conciseness, since nonstandard inertial forces in the moving system description involve the largest dynamic amplification effects, only results arising from the NSA model are proposed. In particular, Figures 11(a) and 11(b) depict the variability of the DAFs for vertical displacement at the center of the arch and at $X_{1} / L=$ 0.75 girder cross section, respectively, as a function of the normalized speed and for several values of the relative bending stiffness ratio; namely, $\varepsilon_{F}=[0.05,0.10,0.15,0.20]$. 


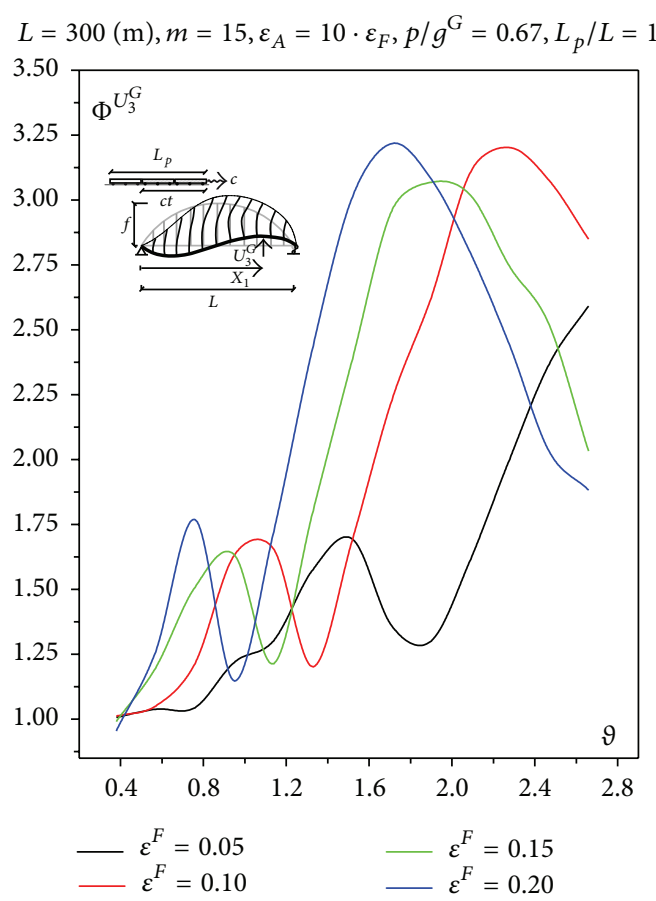

(a)

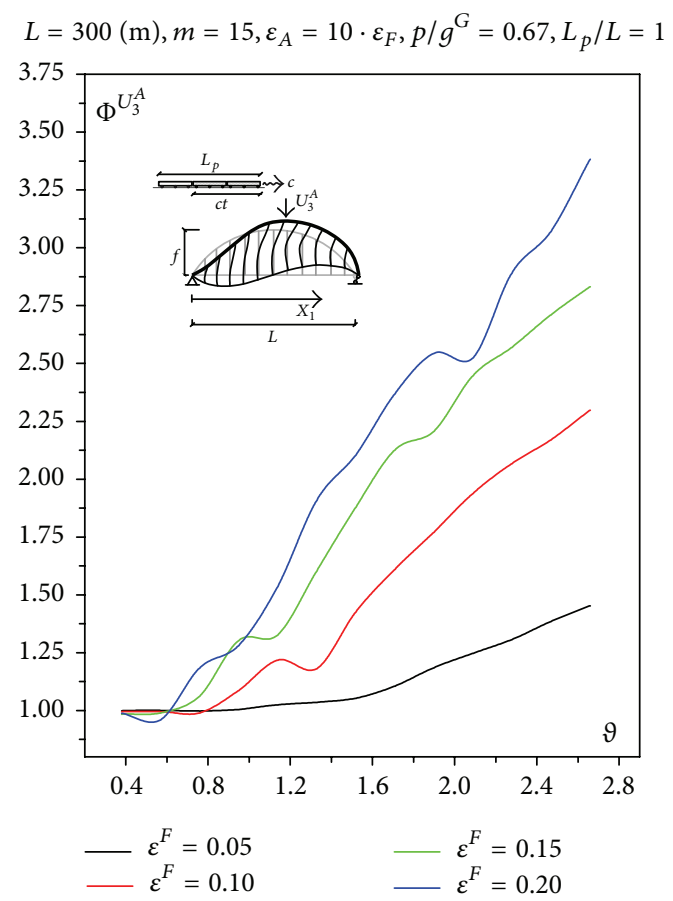

(b)

FIGURE 11: Influence of relative bending stiffness $\left[\varepsilon_{F}\right]$ : variability of DAFs for vertical displacement at $3 / 4$ girder cross section $\left[\Phi^{U_{3}^{G}}\right]($ a) and

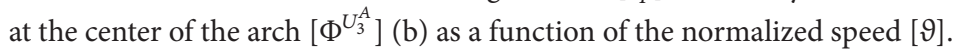

Moreover, in Figures 12(a) and 12(b), DAFs curves of bending moment and axial stress of the girder and the arch profile are reported, respectively. Results indicate that, for low values of bending stiffness ratio $\left(\varepsilon_{F}\right)$, the dynamic amplification of both kinematic and stress variables is strongly reduced. This happens because the arch bridge tends toward a girderdominated scheme, involving deep beams for the girder and a very shallow member for the arch. On the other hand, for increasing values of $\varepsilon_{F}$, a prevailing arch dominated bridge scheme is achieved, in which the girder is basically lighter and more flexible. In such case, the arch bridge is affected by notable dynamic amplification in the medium range of speeds, namely, for $0.6 \leq \vartheta \leq 1.1$, because of resonance issues, as well as in the high range of speeds, in which the effect of nonstandard forces of the moving system arising from Coriolis acceleration and centripetal acceleration becomes quite relevant.

Finally, results are proposed to evaluate the influence of the number of hangers $(m)$ on the dynamic behavior of the bridge. Figures 13 and 14 show the distribution of DAFs for the maximum axial force in hangers and the bending moments along the girder, respectively. The dynamic behavior of the bridge appears to be quite affected by the total number of hangers of the structure.

In particular, the results show that, as far as the total number of hangers increases, the DAFs for both axial force and bending moment tend to be reduced. Such prediction can be explained by the fact that, in the case of a bridge structure with a high number of hangers, the stiffening girder is supported by closely spaced intermediate support, which limits the deformability and, consequently, the internal stress distribution in the structural elements. Moreover, the results denote that, for low range of transit speed of the moving system, the DAFs are quite unaffected by the number of elements in the cable system. As far as the speed of the moving system increases, the influence of the hanger spacing step becomes important, leading to strong amplification in the stresses variables.

\section{Conclusions}

The main aim of the present analysis is to evaluate the dynamic amplification effects on tied-arch bridges produced by moving loads. To this end, a parametric study in terms of dimensionless variables, strictly related to both bridge and moving system characteristics, is developed emphasizing the effects produced by the external mass of the moving system on the dynamic bridge behavior. In particular, the analysis focuses attention on the influence of nonstandard inertial forces involved in the moving system mass description arising from Coriolis acceleration and centripetal acceleration. In order to quantify the amplification effects produced by the moving loads over the static solution, numerical results are proposed in terms of dynamic amplification factors (DAFs). From the analyses, the following conclusions can be drawn:

(i) Extended analyses on DAFs of typical design variable have shown that relevant amplification is observed for 

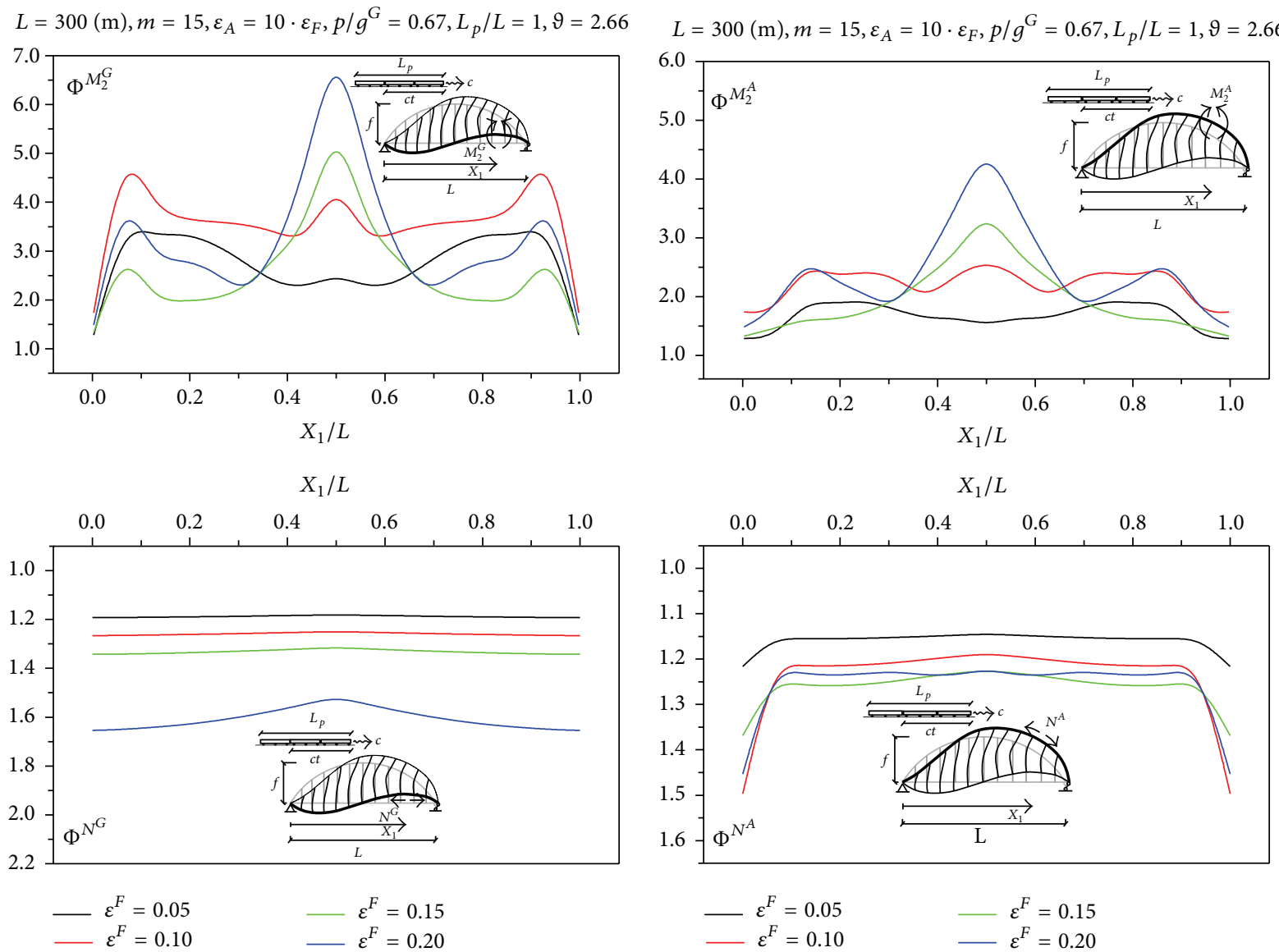

(a)

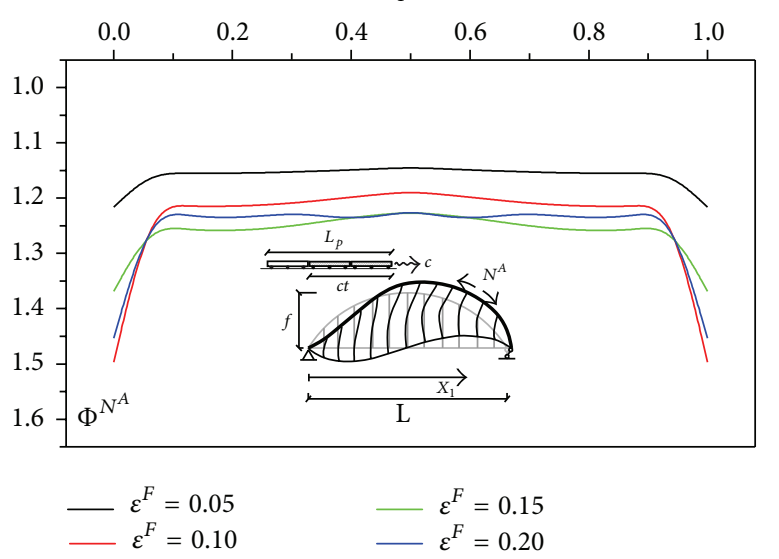

(b)

FIGURE 12: Influence of relative bending stiffness $\left[\varepsilon_{F}\right]$ : DAFs curves for bending moment and axial force of the girder (a) and the arch (b).

TABLE 2: Percentage errors of midspan vertical displacement dynamic amplification factors $\left(\Phi^{U_{3}^{G}}\right)$ between the Moving Force Model (MFM), Standard Analysis (SA), and Nonstandard Analysis (NSA) for different normalized speed parameters ( $\vartheta$ ).

\begin{tabular}{|c|c|c|c|c|c|c|c|c|}
\hline \multirow[t]{2}{*}{$\vartheta$} & \multicolumn{2}{|c|}{$\begin{array}{c}L_{p} / L=0.25 \\
\text { Error }(\%)\end{array}$} & \multicolumn{2}{|c|}{$\begin{array}{c}L_{p} / L=0.50 \\
\text { Error }(\%)\end{array}$} & \multicolumn{2}{|c|}{$\begin{array}{c}L_{p} / L=0.75 \\
\text { Error }(\%)\end{array}$} & \multicolumn{2}{|c|}{$\begin{array}{c}L_{p} / L=1.00 \\
\text { Error }(\%)\end{array}$} \\
\hline & MFM & SA & MFM & SA & MFM & SA & MFM & SA \\
\hline 0.38 & 0.68 & 0.67 & 1.21 & 1.44 & 0.78 & 0.56 & 0.48 & 0.91 \\
\hline 0.57 & 2.03 & 1.18 & 1.84 & 3.23 & 1.36 & 1.78 & 2.14 & 3.53 \\
\hline 0.76 & 10.36 & 8.30 & 4.29 & 6.50 & 3.88 & 5.26 & 2.43 & 3.67 \\
\hline 0.95 & 1.30 & 5.13 & 19.84 & 22.04 & 7.86 & 7.60 & 7.72 & 4.26 \\
\hline 1.14 & 15.25 & 6.28 & 26.24 & 26.53 & 3.86 & 3.17 & 17.53 & 13.46 \\
\hline 1.33 & 15.73 & 7.83 & 1.42 & 6.07 & 20.39 & 11.83 & 11.58 & 10.32 \\
\hline 1.52 & 17.80 & 10.57 & 18.19 & 7.63 & 24.23 & 15.31 & 29.17 & 20.05 \\
\hline 1.71 & 17.15 & 12.97 & 23.97 & 14.07 & 31.85 & 23.08 & 29.81 & 20.82 \\
\hline 1.9 & 20.23 & 17.80 & 25.81 & 17.50 & 32.42 & 25.55 & 31.33 & 18.16 \\
\hline 2.09 & 17.00 & 15.54 & 28.41 & 21.31 & 34.48 & 27.89 & 29.80 & 23.10 \\
\hline 2.28 & 17.36 & 16.20 & 28.77 & 23.69 & 34.49 & 28.48 & 30.05 & 28.94 \\
\hline 2.47 & 17.93 & 15.84 & 30.46 & 25.10 & 35.55 & 29.95 & 34.13 & 31.09 \\
\hline 2.66 & 16.74 & 16.71 & 30.15 & 26.43 & 35.56 & 30.45 & 36.63 & 33.16 \\
\hline
\end{tabular}


$L=300(\mathrm{~m}), \varepsilon_{F}=0.15, \varepsilon_{A}=1.5, p / g^{G}=0.67, L / L_{p}=1$
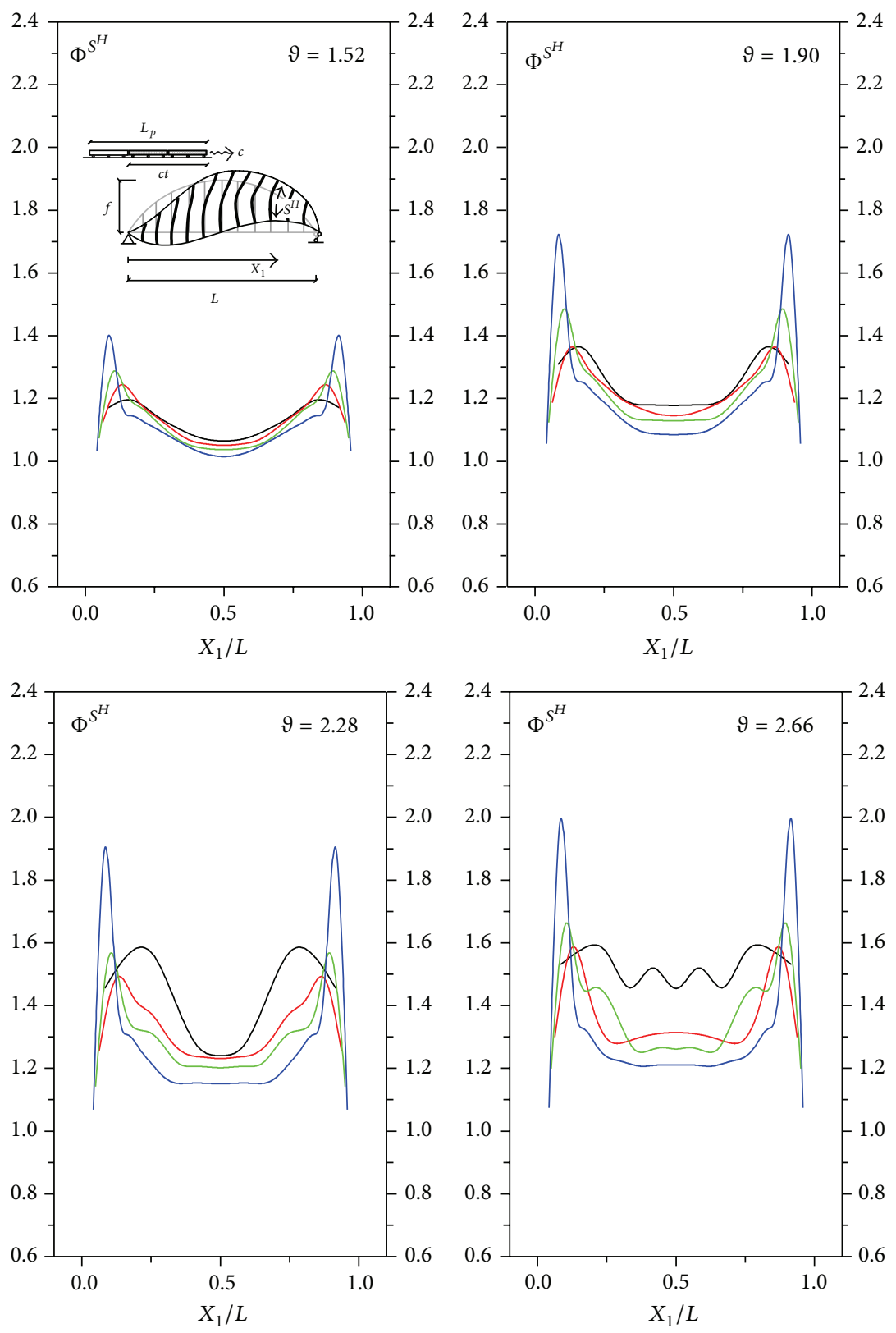

$$
\begin{aligned}
& m=11 \quad-m=19 \\
& -m=15 \quad-m=23
\end{aligned}
$$$$
\begin{aligned}
& -m=11 \quad-m=19 \\
& -m=15 \quad-m=23
\end{aligned}
$$

FIGURE 13: Influence of the number of hangers $[m]$ : DAFs for the maximum axial stress in hangers $\left[\Phi^{S^{H}}\right]$.

kinematic and stress variables, which are much larger than the ones obtained from generalized formula existing in common bridge codes.

(ii) The dynamic behavior of tied-arch bridges appears to be quite dependent from the effect of the travelling mass and large underestimations in dynamic amplification factors are noted if the inertial forces of moving system are not properly evaluated; in particular, the analyses denote that nonstandard inertial forces arising from Coriolis acceleration and centripetal acceleration determine the largest dynamic amplification in both kinematic and stress variables, mainly at high speeds of the moving system.

(iii) Parametric studies in terms of bridge characteristics have shown that that for low values of bending stiffness ratio, that is, in the framework of girderdominated bridge schemes, dynamic amplification 
$L=300(\mathrm{~m}), \varepsilon_{F}=0.15, \varepsilon_{A}=1.5, p / g^{G}=0.67, L / L_{p}=1$
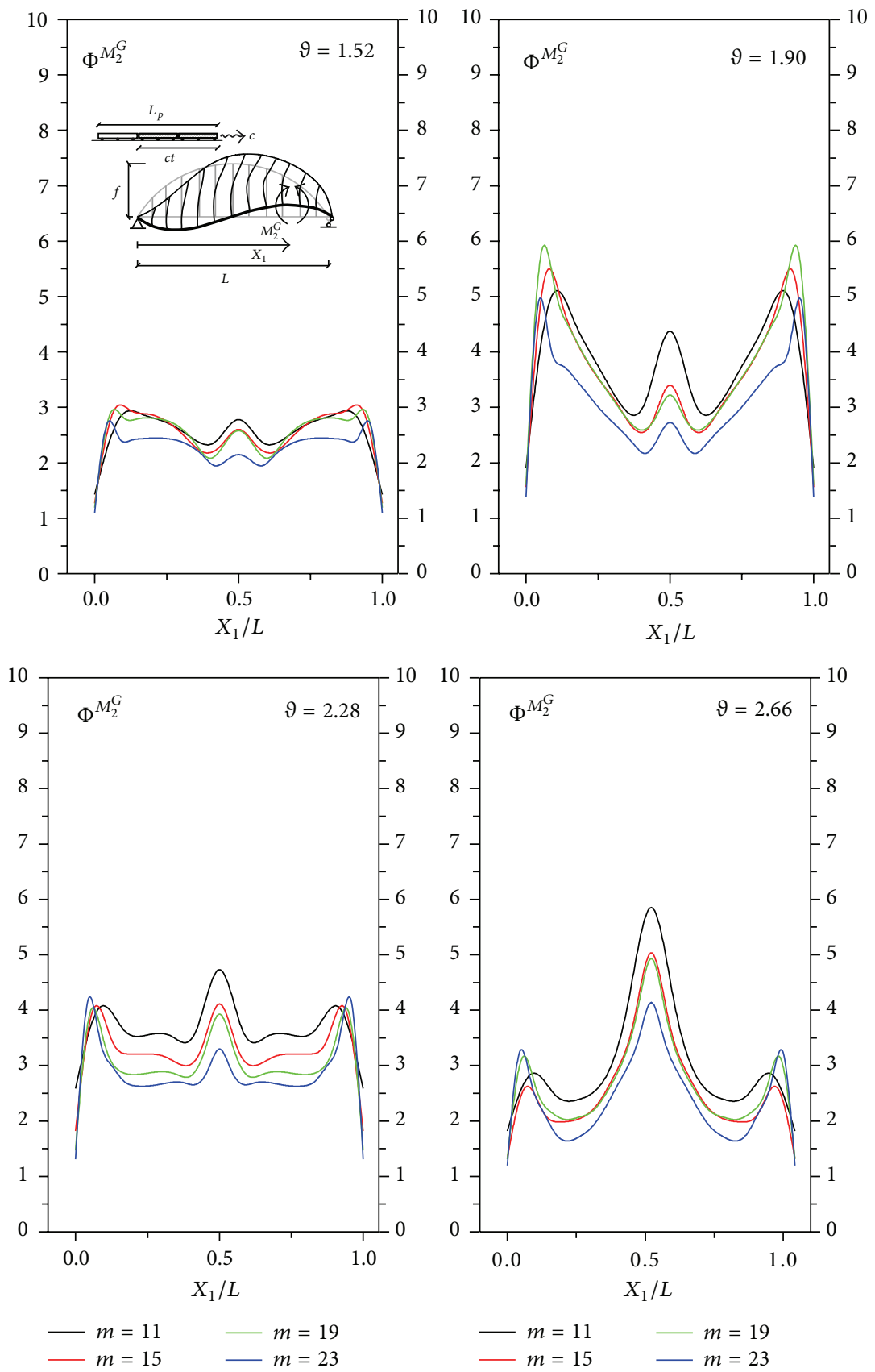

$$
\begin{aligned}
& m=11 \quad-m=19 \\
& -m=15 \quad-m=23
\end{aligned}
$$

FIGURE 14: Influence of the number of hangers $[m]$ : DAFs curve for the bending moment of the girder $\left[\Phi^{M_{2}^{G}}\right]$.

of both kinematic and stress variables is strongly reduced; contrarily, in the case of arch dominated bridge schemes, that is, for large values of relative bridge stiffness, notable dynamic amplification is observed also in medium range of speeds.

(iv) The analyses in terms of hanger discretization have shown that a large number of hangers in the cable system lead to a considerably reduction of the DAFs involved in the bridge especially in the high range of moving system speeds. In this context, several configurations and scenarios have been investigated, emphasizing the influence of DAFs of typical design variables on the number of hangers adopted in the definition of the cable system.

\section{Appendix}

The analytic description of the moving mass function $\lambda$, acting on the girder profile, is defined as

$$
\lambda=\lambda\left(X_{1}, t\right)=\lambda_{\mathrm{ML}} \bar{H}\left(X_{1}+L_{p}-c t\right) \bar{H}\left(c t-X_{1}\right),
$$


where $\bar{H}(\cdot)$ is the Heaviside step function, $L_{p}$ is the length of the moving loads, and $\lambda_{\mathrm{ML}}$ is the mass linear density of the moving system. Moreover, the expression of the moving loads, for a fixed inertial reference frame $\left(0,{\underset{\sim}{1}}_{1}, \underline{\sim}_{2}, \underline{n}_{3}\right)$, is defined by the weight and the inertial forces produced by the inertial characteristics and the unsteady mass distribution of the moving loads, as follows:

$$
\begin{aligned}
p_{X_{i}} & =\lambda g n_{\sim} \times{\underset{\sim}{3}}_{3}+\frac{d}{d t}\left[\lambda \frac{d \bar{U}_{i}^{m}}{d t}\right] \\
& =\lambda g n_{\sim} \times{\underset{\sim}{3}}_{3}+\frac{d \lambda}{d t} \frac{d \bar{U}_{i}^{m}}{d t}+\lambda \frac{d^{2} \bar{U}_{i}^{m}}{d t^{2}},
\end{aligned}
$$

where $\bar{U}^{m}$ is the moving load kinematic which can be expressed as a function of the displacement and rotation fields of the centroid axis of the girder, that is, $\left(U_{1}^{G}, U_{2}^{G}, U_{3}^{G}\right)$ and $\left(\Psi_{1}^{G}, \Psi_{2}^{G}, \Psi_{3}^{G}\right)$ :

$$
\begin{aligned}
\bar{U}_{1}^{m}\left(X_{1}, X_{2}, X_{3}, t\right) & =U_{1}^{G}\left(X_{1}, t\right)-\Psi_{3}^{G}\left(X_{1}, t\right) e, \\
\bar{U}_{2}^{m}\left(X_{1}, t\right) & =U_{2}^{G}\left(X_{1}, t\right), \\
\bar{U}_{3}^{m}\left(X_{1}, X_{2}, X_{3}, t\right) & =U_{3}^{G}\left(X_{1}, t\right)+\Psi_{1}^{G}\left(X_{1}, t\right) e .
\end{aligned}
$$

Since the external forces, defined by (A.2), are described in terms of a moving coordinate, the time dependent description introduces the following expressions for the velocity and the acceleration functions:

$$
\begin{aligned}
\frac{d \bar{U}_{i}^{m}}{d t} & =\frac{\partial \bar{U}_{i}^{m}}{\partial t}+\frac{\partial \bar{U}_{i}^{m}}{\partial X_{1}} \frac{\partial X_{l}(t)}{\partial t} \\
\frac{d^{2} \bar{U}_{i}^{m}}{d t^{2}} & =\frac{\partial^{2} \bar{U}_{i}^{m}}{\partial t^{2}}+2 c \frac{\partial^{2} \bar{U}_{i}^{m}}{\partial t \partial X_{1}}+c^{2} \frac{\partial^{2} \bar{U}_{i}^{m}}{\partial X_{1}^{2}}
\end{aligned}
$$

where the second and the third terms on right hand side in the acceleration function are known in the literature as the Coriolis acceleration and centripetal acceleration, respectively [34]. Finally, on the basis of (A.1)-(A.4) assuming that the mass does not separate from the beam during its horizontal and vertical vibrations, the external load functions are defined by the following relationships:

$$
\begin{aligned}
p_{X_{1}}= & \lambda \frac{\partial^{2} U_{1}^{G}}{\partial t^{2}}+\frac{d \lambda}{d t} \frac{\partial U_{1}^{G}}{\partial t} \\
p_{X_{2}}= & \lambda \frac{\partial^{2} U_{2}^{G}}{\partial t^{2}}+\frac{d \lambda}{d t} \frac{\partial U_{2}^{G}}{\partial t} \\
p_{X_{3}}= & \lambda\left[g+\frac{\partial^{2} U_{3}^{G}}{\partial t^{2}}\right]+\frac{d \lambda}{d t}\left[\frac{\partial U_{3}^{G}}{\partial t}+c \frac{\partial U_{3}^{G}}{\partial X_{1}}\right] \\
& +\lambda\left[2 c \frac{\partial^{2} U_{3}^{G}}{\partial t \partial X_{1}}+c \frac{\partial^{2} U_{3}^{G}}{\partial X_{1}^{2}}\right] .
\end{aligned}
$$

Moreover, the derivation of the explicit expressions of the finite element matrixes reported in (4) can be obtained by using the discrete approximation defined by (3) as follows:

$$
\begin{aligned}
& {\left[M_{n s}\right]_{h k}=\int_{i-1}^{i+1} \lambda N_{h} N_{k} d X_{1},} \\
& {\left[C_{n s}\right]_{h k}} \\
& =\int_{i-1}^{i+1}\left[\frac{d \lambda}{d t} N_{h} N_{k}+2 \lambda c \frac{d \lambda}{d t} \Xi_{1} \frac{\partial N_{h}}{\partial X_{1}} N_{k}\right] d X_{1} \\
& {\left[K_{n s}\right]_{h k}=c \int_{i-1}^{i+1}\left[\lambda \frac{\partial^{2} N_{h}}{\partial X_{1}^{2}} N_{k}+\frac{d \lambda}{d t} \frac{\partial N_{h}}{\partial X_{1}} N_{k}\right] d X_{1} .}
\end{aligned}
$$

\section{Competing Interests}

The authors declare that there are no competing interests regarding the publication of this paper.

\section{References}

[1] C. Pellegrino, G. Cupani, and C. Modena, "The effect of fatigue on the arrangement of hangers in tied arch bridges," Engineering Structures, vol. 32, no. 4, pp. 1140-1147, 2010.

[2] L. Fryba, Vibration of Solids and Structures under Moving Loads, Thomas Telford, Prague, Czech Republic, 1999.

[3] S. P. Timoshenko and D. H. Young, Theory of Structures, McGraw-Hill, New York, NY, USA, 2nd edition, 1965.

[4] S. P. Timoshenko, "On the forced vibration of bridges," Philosophical Magazine, vol. 43, no. 257, pp. 1018-1019, 1922.

[5] Y.-B. Yang, J.-D. Yau, and L.-C. Hsu, "Vibration of simple beams due to trains moving at high speeds," Engineering Structures, vol. 19, no. 11, pp. 936-943, 1997.

[6] H. Xia, Y. L. Xu, and T. H. T. Chan, "Dynamic interaction of long suspension bridges with running trains," Journal of Sound and Vibration, vol. 237, no. 2, pp. 263-280, 2000.

[7] F. T. K. Au, J. J. Wang, and Y. K. Cheung, "Impact study of cable-stayed bridge under railway traffic using various models," Journal of Sound and Vibration, vol. 240, no. 3, pp. 447-465, 2001.

[8] F. T. K. Au, J. J. Wang, and Y. K. Cheung, "Impact study of cable-stayed railway bridges with random rail irregularities," Engineering Structures, vol. 24, no. 5, pp. 529-541, 2002.

[9] D. Bruno, F. Greco, and P. Lonetti, "Dynamic impact analysis of long span cable-stayed bridges under moving loads," Engineering Structures, vol. 30, no. 4, pp. 1160-1177, 2008.

[10] X. Lei and N.-A. Noda, "Analyses of dynamic response of vehicle and track coupling system with random irregularity of track vertical profile," Journal of Sound and Vibration, vol. 258, no. 1, pp. 147-165, 2002.

[11] Y.-B. Yang, S.-S. Liao, and B.-H. Lin, "Impact formulas for vehicles moving over simple and continuous beams," Journal of Structural Engineering, vol. 121, no. 11, pp. 1644-1650, 1995.

[12] F. Lu, J. H. Lin, D. Kennedy, and F. W. Williams, "An algorithm to study non-stationary random vibrations of vehicle-bridge systems," Computers and Structures, vol. 87, no. 3-4, pp. 177-185, 2009.

[13] H. Xia and N. Zhang, "Dynamic analysis of railway bridge under high-speed trains," Computers and Structures, vol. 83, no. 23-24, pp. 1891-1901, 2005. 
[14] F. Greco, P. Lonetti, and A. Pascuzzo, "Dynamic analysis of cable-stayed bridges affected by accidental failure mechanisms under moving loads," Mathematical Problems in Engineering, vol. 2013, Article ID 302706, 20 pages, 2013.

[15] P. Lonetti and A. Pascuzzo, "Vulnerability and failure analysis of hybrid cable-stayed suspension bridges subjected to damage mechanisms," Engineering Failure Analysis, vol. 45, pp. 470-495, 2014.

[16] J. M. Rocha, A. A. Henriques, and R. Calçada, "Safety assessment of a short span railway bridge for high-speed traffic using simulation techniques," Engineering Structures, vol. 40, pp. 141154, 2012.

[17] J. M. Rocha, A. A. Henriques, and R. Calçada, "Probabilistic safety assessment of a short span high-speed railway bridge," Engineering Structures, vol. 71, pp. 99-111, 2014.

[18] P. K. Chatterjee and T. K. Datta, "Dynamic analysis of arch bridges under travelling loads," International Journal of Solids and Structures, vol. 32, no. 11, pp. 1585-1594, 1995.

[19] J.-S. Wu and L.-K. Chiang, "Dynamic analysis of an arch due to a moving load," Journal of Sound and Vibration, vol. 269, no. 3-5, pp. 511-534, 2004.

[20] D. Huang, "Dynamic and impact behavior of half-through arch bridges," Journal of Bridge Engineering, vol. 10, no. 2, pp. 133-141, 2005.

[21] S.-H. Ju and H.-T. Lin, "Numerical investigation of a steel arch bridge and interaction with high-speed trains," Engineering Structures, vol. 25, no. 2, pp. 241-250, 2003.

[22] W. Lacarbonara and V. Colone, "Dynamic response of arch bridges traversed by high-speed trains," Journal of Sound and Vibration, vol. 304, no. 1-2, pp. 72-90, 2007.

[23] J.-R. Yang, J.-Z. Li, and Y.-H. Chen, "Vibration analysis of CFST tied-arch bridge due to moving vehicles," Interaction and Multiscale Mechanics, vol. 3, no. 4, pp. 389-403, 2010.

[24] P. H. Wang, T. C. Tseng, and C. G. Yang, "Initial shape of cablestayed bridges," Computers and Structures, vol. 46, no. 6, pp. 1095-1106, 1993.

[25] P.-H. Wang, T.-Y. Tang, and H.-N. Zheng, "Analysis of cablestayed bridges during construction by cantilever methods," Computers and Structures, vol. 82, no. 4-5, pp. 329-346, 2004.

[26] N. J. Gimsing and C. T. Georgakis, Cable Supported Bridges. Concept and Design, John Wiley \& Sons, 3rd edition, 2012.

[27] P. Lonetti and A. Pascuzzo, "Design analysis of the optimum configuration of self-anchored cable-stayed suspension bridges," Structural Engineering and Mechanics, vol. 51, no. 5, pp. 847-866, 2014.

[28] D. Bruno, P. Lonetti, and A. Pascuzzo, "An optimization model for the design of network arch bridges," Computers \& Structures, vol. 170, pp. 13-25, 2016.

[29] P. Wriggers, Nichtlineare Finite-Element-Methoden, Springer, Hanover, Germany, 1st edition, 2001.

[30] COMSOL, Comsol Multiphysics Reference Manual, COMSOL, Burlington, Mass, USA, 2010.

[31] A. W. Hedgren, R. Brockenbrough, and F. S. Merritt, "Arch bridges," in Structural Steel Designer's Handbook, Access Engineering, McGraw Hill, New York, NY, USA, 5th edition, 2011.

[32] European Committee for Standardization, Eurocode 1: Actions on Structures. Part 3: Traffic Loads on Bridges, 2002.

[33] European Committee for Standardisation, Eurocode 3: Design of Steel Structures. Part 2: Steel Bridges, 2006.
[34] L. Kwasniewski, H. Li, J. Wekezer, and J. Malachowski, "Finite element analysis of vehicle-bridge interaction," Finite Elements in Analysis and Design, vol. 42, no. 11, pp. 950-959, 2006. 


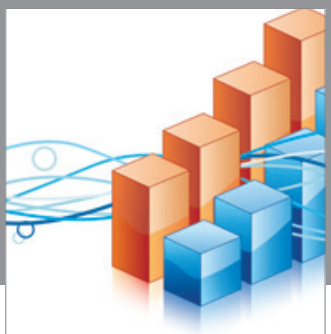

Advances in

Operations Research

vatem alat4

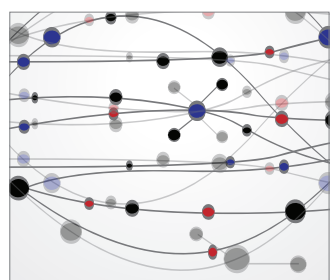

\section{The Scientific} World Journal
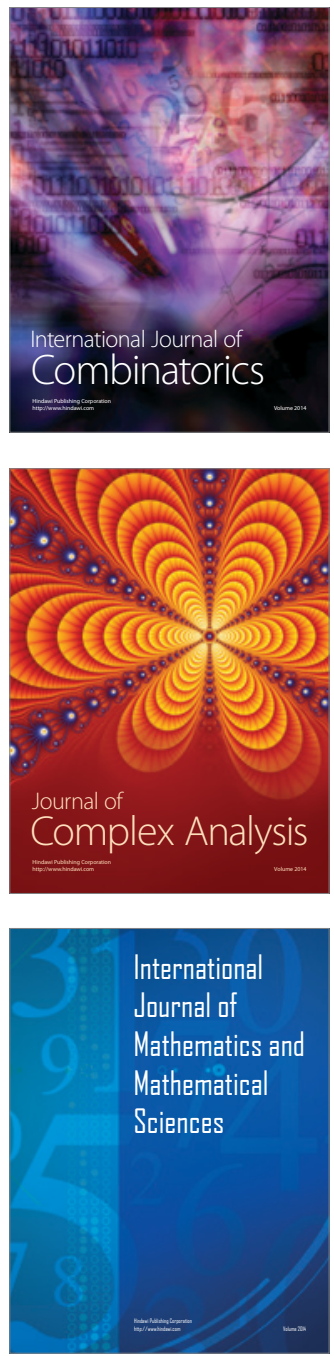
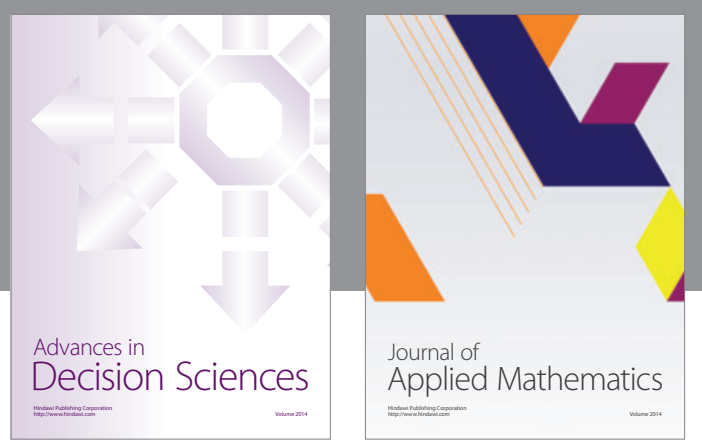

Algebra

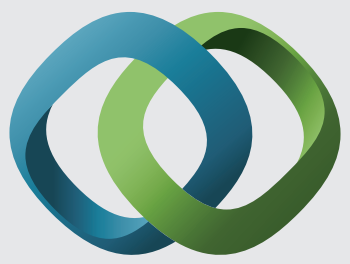

\section{Hindawi}

Submit your manuscripts at

http://www.hindawi.com
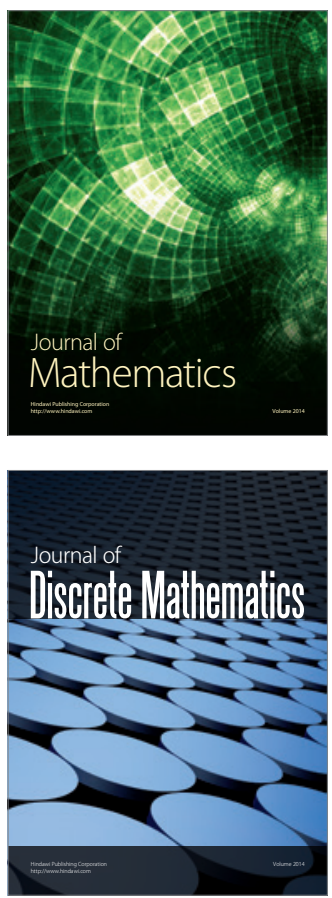

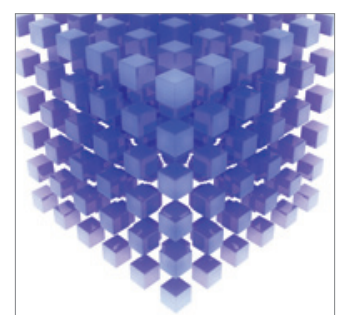

Mathematical Problems in Engineering
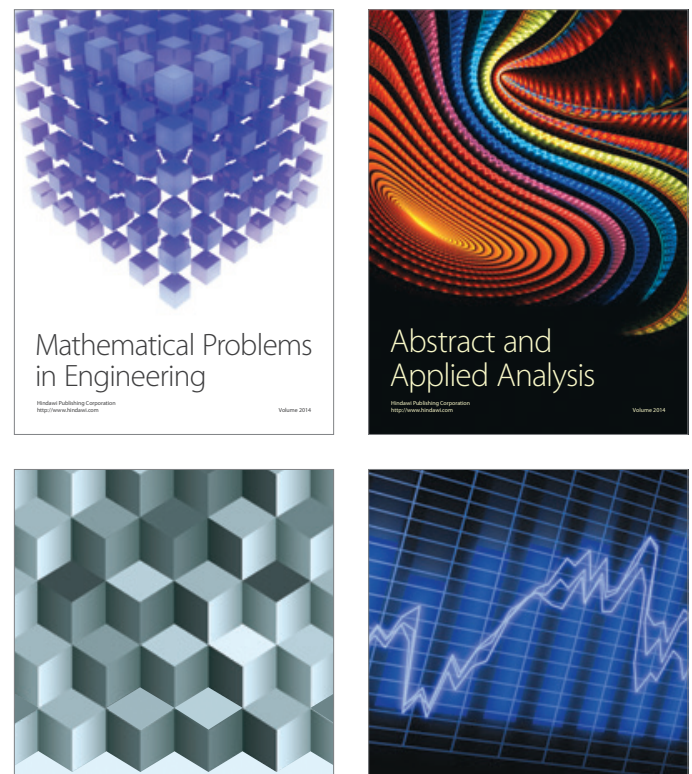

Journal of

Function Spaces

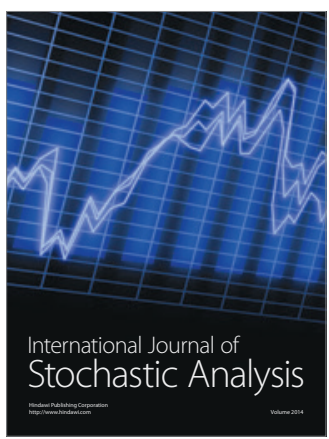

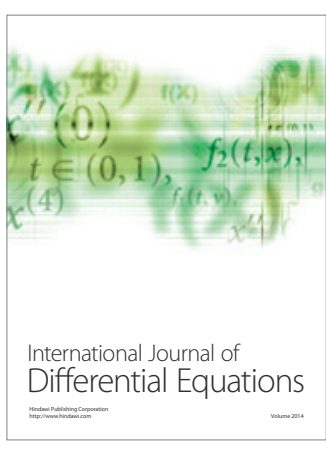
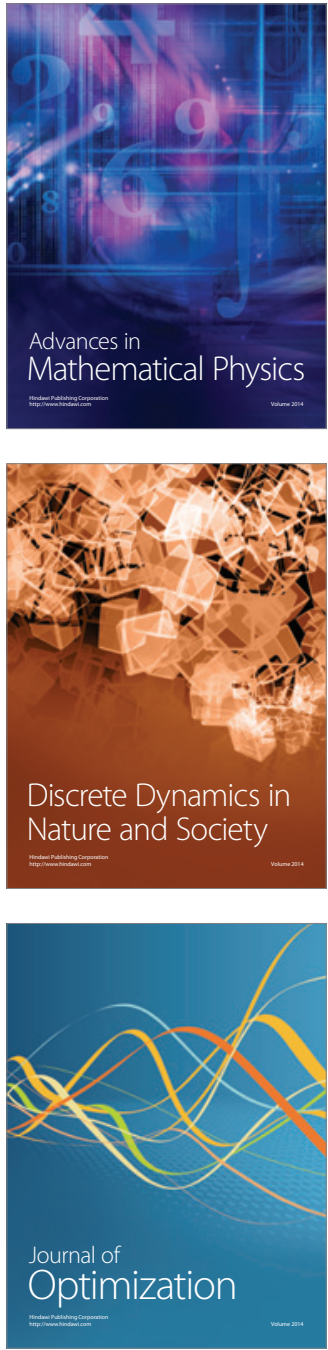